\title{
Allele-specific RNA interference prevents neuropathy in Charcot-Marie-Tooth disease type 2D mouse models
}

\author{
Kathryn H. Morelli, ${ }^{1,2}$ Laurie B. Griffin, ${ }^{3,4}$ Nettie K. Pyne, ${ }^{5}$ Lindsay M. Wallace, ${ }^{5}$ Allison M. Fowler, ${ }^{5}$ Stephanie N. Oprescu, ${ }^{6}$ \\ Ryuichi Takase, ${ }^{7}$ Na Wei, ${ }^{8}$ Rebecca Meyer-Schuman, ${ }^{6}$ Dattatreya Mellacheruvu, ${ }^{9,10}$ Jacob O. Kitzman, ${ }^{6}$ Samuel G. Kocen, ${ }^{1}$ \\ Timothy J. Hines, ${ }^{1}$ Emily L. Spaulding, ${ }^{1,2}$ James R. Lupski, ${ }^{11,12,13}$ Alexey Nesvizhskii, ${ }^{9,10}$ Pedro Mancias, ${ }^{14}$ Ian J. Butler, ${ }^{14}$ \\ Xiang-Lei Yang, ${ }^{8}$ Ya-Ming Hou, ${ }^{7}$ Anthony Antonellis, ${ }^{3,6}$ Scott Q. Harper, ${ }^{5,15}$ and Robert W. Burgess ${ }^{1,2}$
}

'The Jackson Laboratory, Bar Harbor, Maine, USA. ${ }^{2}$ Graduate School of Biomedical Science and Engineering, University of Maine, Orono, Maine, USA. ${ }^{3 P r o g r a m}$ in Cellular and Molecular Biology, and ${ }^{4}$ Medical Scientist Training Program, University of Michigan, Ann Arbor, Michigan, USA. ${ }^{5}$ Center for Gene Therapy, The Research Institute at Nationwide Children's Hospital, Columbus, Ohio, USA. ${ }^{6}$ Department of Human Genetics, University of Michigan, Ann Arbor, Michigan, USA. `Department of Biochemistry and Molecular Biochemistry, Thomas Jefferson University, Philadelphia, Pennsylvania, USA. ${ }^{8}$ Department of Molecular Medicine, The Scripps Research Institute, La Jolla, California, USA. 'Department of Pathology, and ${ }^{10}$ Department of Computational Medicine and Bioinformatics, University of Michigan, Ann Arbor, Michigan, USA. "Department of Molecular and Human Genetics, and ${ }^{12}$ Department of Pediatrics, Baylor College of Medicine, Houston, Texas, USA. ${ }^{13}$ Texas Children's Hospital, Houston, Texas, USA. ${ }^{14}$ Department of Pediatrics, McCovern Medical School, The University of Texas Health Science Center at Houston, and Children's Memorial Hermann Hospital, Houston, Texas, USA. ${ }^{15}$ Department of Pediatrics, The Ohio State University College of Medicine, Columbus, Ohio, USA.

\begin{abstract}
Gene therapy approaches are being deployed to treat recessive genetic disorders by restoring the expression of mutated genes. However, the feasibility of these approaches for dominantly inherited diseases - where treatment may require reduction in the expression of a toxic mutant protein resulting from a gain-of-function allele - is unclear. Here we show the efficacy of allele-specific RNAi as a potential therapy for Charcot-Marie-Tooth disease type 2D (CMT2D), caused by dominant mutations in glycyl-tRNA synthetase (GARS). A de novo mutation in GARS was identified in a patient with a severe peripheral neuropathy, and a mouse model precisely recreating the mutation was produced. These mice developed a neuropathy by 3-4 weeks of age, validating the pathogenicity of the mutation. RNAi sequences targeting mutant GARS mRNA, but not wildtype, were optimized and then packaged into AAV9 for in vivo delivery. This almost completely prevented the neuropathy in mice treated at birth. Delaying treatment until after disease onset showed modest benefit, though this effect decreased the longer treatment was delayed. These outcomes were reproduced in a second mouse model of CMT2D using a vector specifically targeting that allele. The effects were dose dependent, and persisted for at least 1 year. Our findings demonstrate the feasibility of AAV9-mediated allele-specific knockdown and provide proof of concept for gene therapy approaches for dominant neuromuscular diseases.
\end{abstract}

\section{Introduction}

Personalized medicine seeks to provide optimized treatments for individuals based on the molecular characteristics of their specific disease. For example, cancer treatments are customized to the genetics of the patient and the tumor $(1,2)$. However, developing personalized treatments for rare Mendelian disorders is challenging, and only about $5 \%$ of the more than 6800 recognized rare diseases have approved therapies (https:// www.genome.gov/27531963/faq-about-rare-diseases/). Often, even when a disease gene is defined, the pathological impacts of specific genetic lesions may be uncertain or variable. In such cases, animal models recapitulating molecular lesions offer a means for validating the causality of new human mutations, and can serve as surrogates in preclinical therapeutic development $(3,4)$.

Conflict of interest: The authors have declared that no conflict of interest exists. Copyright: () 2019, American Society for Clinical Investigation.

Submitted: May 24, 2019; Accepted: September 10, 2019; Published: November 11, 2019 Reference information: / Clin Invest. 2019;129(12):5568-5583.

https://doi.org/10.1172/JCI130600.
Gene therapy is a potentially powerful approach for treating rare genetic disorders, as it can precisely address the primary cause of these conditions (5). Gene therapy approaches are now in the clinic to treat recessive loss-of-function conditions by restoring the expression of the mutated genes $(6,7)$. However, the feasibility of gene silencing therapy to treat dominantly inherited, monoallelic mutations - where treatment may require reduction in the expression of a toxic mutant protein resulting from a gain-of-function allele - is unclear. To date, ClinicalTrials.gov lists only 2 phase I/II studies designed to test the safety of vector-expressed inhibitory RNAs in humans, and both were indicated for suppressing chronic hepatitis virus infection (NCT01899092 and NCT02315638). Thus, RNAibased therapy is still an emerging field.

Here, we combine precision models of a dominant inherited neuropathy - Charcot-Marie-Tooth disease type 2D (MIM \#601472), caused by mutations in glycyl-tRNA synthetase (GARS) (MIM \#600287) - with allele-specific knockdown by viral vectordelivered gene therapy to demonstrate the effectiveness of this strategy. We designed our treatment for a single patient, but this sets an important precedent, as $90 \%$ of inherited peripheral 
neuropathies - as well as the majority of familial amyotrophic lateral sclerosis cases - are inherited in an autosomal dominant manner $(8,9)$.

Charcot-Marie-Tooth disease type 2D (CMT2D) is a progressive, inherited axonal neuropathy caused by dominant mutations in GARS, encoding glycyl-tRNA synthetase (10). Mutations in GARS also cause a purely motor neuropathy, clinically designated as distal spinal muscular atrophy type $\mathrm{V}$, but this is allelic with CMT2D (11). There is no treatment for CMT2D or any other form of inherited peripheral neuropathy. To date, at least 19 individual mutations in GARS have been identified in patients with CMT2D (12), all of which result in single-amino acid changes in different functional domains of GARS (10, 13-16). However, the mechanisms through which mutant forms of GARS cause axon degeneration remain unclear, limiting the development of a small-molecule therapy.

Most disease-associated GARS variants cause impaired enzymatic activity in the charging of glycine onto tRNA ${ }^{\text {Gly }}$ in vitro and/ or decreased cellular viability in yeast complementation assays, consistent with a loss-of-function effect $(17,18)$. However, proteinnull alleles in mice and humans do not cause dominant neuropathy, ruling out haploinsufficiency and suggesting a dominantnegative (antimorph) mechanism (19-22). Furthermore, transgenic overexpression of wild-type (WT) GARS does not rescue the neuropathy in mouse models, suggesting that mutant forms of GARS adopt a toxic gain-of-function (neomorph) activity that the WT protein cannot outcompete (20). One proposed neomorphic mechanism involves the abnormal binding of mutant GARS to the developmental receptor neuropilin-1 (NRP1). This interaction competes with the normal binding of vascular endothelial growth factor (VEGF), an endogenous ligand of NRP1 (23).

Together, these data support a model in which suppression of the mutant allele of GARS should be of therapeutic benefit, whereas enhancing normal GARS function is ineffective. To achieve this suppression, we developed a gene therapy strategy to reduce the levels of mutant Gars transcripts through allele-specific RNAi, triggered through the delivery of mutant Gars-targeted artificial microRNA (miRNA) expression cassettes packaged within self-complementary adeno-associated viral vectors, serotype 9 (scAAV9).

\section{Results}

We sought to demonstrate the therapeutic efficacy of a patientspecific RNAi-based gene therapy in a "humanized" mouse model expressing a patient-specific GARS mutation introduced into the mouse Gars gene. A 13-month-old female presented with impaired motor skills and regressing motor milestones involving both upper and lower extremities. She was able to sit independently, but used her arms to stabilize herself in a sitting position (tripod sitting). Increased lumbar lordosis was also noted at first examination. Dysmorphic features were noted, likely due to generalized muscle atrophy. Extraocular muscle function was normal. Deep tendon reflexes were difficult to obtain or absent, and she showed general, marked decreases in muscle tone, head lag, axillary slippage, mild tongue atrophy, ligamentous laxity in the hands and feet, and excessive retraction of the chest wall. The patient was delivered by $\mathrm{C}$-section at 37 weeks gestation after a pregnancy complicated by hypertension. She required oxygen supplementation and had mild neonatal jaundice, but was discharged after 5 days. Newborn screening was normal, and motor development was probably normal at first, with the ability to reach for objects at 4 months and stand with support at 8 months. There was no history of seizures, and cognitive development was uncompromised.

Muscle biopsy at 15 months was indicative of neurogenic changes consistent with motor neuronopathy or neuropathy. This included marked atrophy of type I and II fibers with isolated, clustered, and fascicles of hypertrophied type I myofibers. There was no evidence of myofiber necrosis, degeneration, or regeneration, nor of dystrophic or inflammatory myopathy. Electromyography and nerve conduction studies were consistent with motor neuron disease: motor nerve conduction velocities were reduced $(26 \mathrm{~m} / \mathrm{s}$ upper and $15 \mathrm{~m} / \mathrm{s}$ lower), while sensory examination revealed no deficits, including sensory nerve conduction (2.0 milliseconds latency and $46 \mu \mathrm{V}$ at her wrist). At 20 months, MRI of the brain and cervical spinal cord were normal, as was an analysis of the cerebrospinal fluid. She did not display evidence of further decline and did not regress in any areas. Indeed, she seemed slightly stronger overall with no problems swallowing or drinking. Cranial nerves were intact and there was no evidence of tongue fasciculation. However, motor examination revealed decreased muscle mass and tone, axillary slippage, flaccid lower limbs, and higharched feet. She could still sit independently using her upper limbs for support, and displayed head lag and an inability to stand on her own. A diagnosis of neuronopathy was suspected; however, there was no reported family history of this phenotype, and the patient's nonidentical twin brother was unaffected.

A recessive or dominant de novo inheritance pattern was predicted. Patient DNA was extracted and subjected to both targeted and unbiased screening strategies. This revealed 2 full-length, WT copies of the SMN1 gene and 1 copy of SMN2, ruling out spinal muscular atrophy types I through III. Furthermore, targeted analysis did not reveal mutations in IGHMBP2 or UBE1, ruling out recessive, distal spinal muscular atrophy and X-linked spinal muscular atrophy type II, respectively. Whole-exome sequencing analysis revealed that the patient is heterozygous for a 12-nucleotide deletion in exon 8 of the glycyl-tRNA synthetase (GARS) gene (c.894_905del; GenBank NM_002047.2). This mutation resulted in the deletion of 4 amino acids in the GARS protein (p.Glu299_Gln302del; NP_002038.2), hereafter referred to as $\triangle$ ETAQ. No other potentially pathogenic variant was identified at another locus that could potentially explain the severity of the neuropathy by a dual molecular diagnosis (24). Neither parent carries the identified GARS mutation, nor does the patient's twin brother, indicating a de novo mutation. GARS functions to ligate glycine onto cognate tRNA molecules. The $\triangle E T A Q G A R S$ mutation results in the deletion of 4 amino acid residues that are conserved from human to bacteria and that reside within the glycine-binding pocket (Figure $1 \mathrm{~A}$ and ref. 25).

To determine whether the $\triangle \mathrm{ETAQ}$ GARS mutation affects mRNA expression or stability, we performed RNA-Seq in patient primary dermal fibroblasts. These analyses revealed an even distribution of WT (53.7\%) and $\triangle E T A Q(46.3 \%)$ RNA-Seq reads, indicating that $\triangle \mathrm{ETAQ} G A R S$ does not dramatically affect transcript 
A

H. sapiens FIGPGGNMPGYLRPETAQGIFLNF M. musculus FIGPGGNMPGYLRPETAQGIFLNF

D. rerio SIGPGGNMQGYLRPETAQGIFLNF D. melanogaster QIGPTGLVKGFLRPETAQGIFVNF C. elegans QIGPTGDFKAFLRPETAQGIFVNF S. cerevisiae AIGPSGQLKGYLRPETAQGQFLNF E. coli GASADSAMDLYLRPETAQGIFVNF

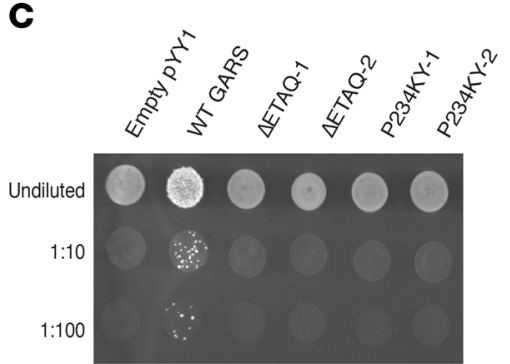

B

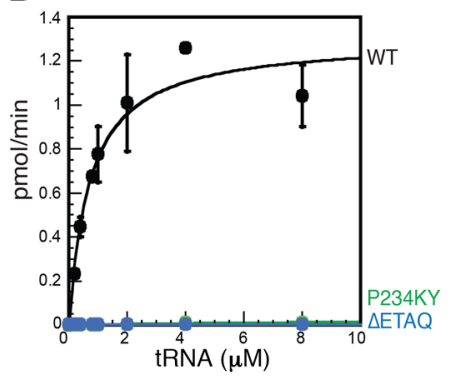

Figure 1. In vitro characterization of $\triangle E T A Q$ mutation. (A) The position and evolutionary conservation of the $\triangle \mathrm{ETAQ}$ (red) and P234KY (green) GARS mutations. (B) Initial aminoacylation rates (pmol/min) of WT (black), P234KY (green), and $\triangle E T A Q$ (blue) GARS were plotted against tRNA concentrations and fit to the Michaelis-Menten equation. (C) Representative cultures of yeast strains lacking GRS1 to test for growth in the presence of each mutation ( $\triangle E T A Q$ or $\mathrm{P} 234 \mathrm{KY}$ ) modeled in the human GARS open reading frame. (D) WT, P234KY, or $\triangle E T A Q$ GARS was expressed (with a V5 tag) and tested by immunoprecipitation with an anti-NRP1 antibody to detect aberrant interactions. Western blots were performed with anti-NRP1 and anti-V5 antibodies.

D

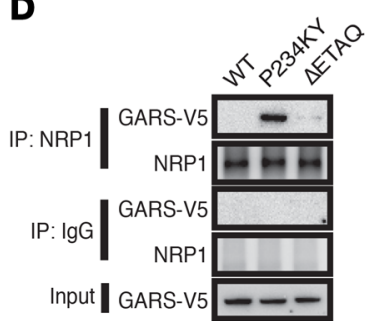
Immunoprecipitation (IP), negative control (IgG), and input experiments are indicated. levels (Supplemental Figure 1A; supplemental material available online with this article; https://doi.org/10.1172/JCI130600DS1). To examine GARS protein levels, we performed Western blot analysis on whole-cell lysates from patient cells compared with a control primary dermal fibroblast cell line (i.e., bearing no GARS mutations). These experiments did not reveal an observable difference in total GARS protein levels in the affected fibroblasts compared with the control cell line (Supplemental Figure 1B). We next performed aminoacylation assays and yeast complementation tests to assess whether $\triangle \mathrm{ETAQ} G A R S$ affects the primary activity of the enzyme. Analysis of the initial rate of aminoacylation as a function of the tRNA substrate concentration showed that $\triangle$ ETAQ GARS retained less than $0.01 \%$ aminoacylation activity (Figure 1B and Supplemental Table 1) compared with WT GARS. In parallel, we tested the previously described mouse allele $\mathrm{P} 234 \mathrm{KY}$ ( $\mathrm{P} 278 \mathrm{KY}$ in the mouse, where 234 is numbered without the 44-amino acid mitochondrial targeting sequence), given its nearby location in the protein (Figure $1 \mathrm{~A}$ and ref. 22). Although the P234KY allele showed activity in assays with saturating tRNA and glycine substrate concentrations (22), a reevaluation of kinetic properties under Michaelis-Menten conditions showed a marked decrease in enzyme activity (Figure 1B and Supplemental Table 1). The reduced function of the $\triangle E T A Q$ allele was further supported by the failure of this mutant protein to complement ablated cellular growth associated with deletion of the yeast ortholog GRS1 (Figure 1C). Data from this latter assay also support the loss-offunction effect associated with P278KY Gars (Figure 1C), and are consistent with the failure of the mouse P278KY allele to complement an RNA-null allele of Gars (22).

Neuropathy-associated GARS mutations bind inappropriately to NRP1 (23). To test for binding between $\triangle$ ETAQ GARS and NRP1, we expressed V5-tagged WT, P234KY, and $\triangle$ ETAQ GARS in the mouse NSC-34 cells. After immunoprecipitation with an anti-NRP1 antibody, Western blots were performed using an anti-V5 antibody. In contrast to the reported strong association with $\mathrm{P} 234 \mathrm{KY}$
GARS (23), the V5 signal associated with $\triangle$ ETAQ GARS was much weaker, although stronger than that of WT GARS, which showed no V5 signal (Figure 1D). No interaction between $\triangle E T A Q$ GARS and NRP1 was detected in unbiased mass spectrometry analyses of proteins immunoprecipitated from mouse neuroblastoma (MN1) cells expressing FLAG-tagged WT or V5-tagged $\triangle$ ETAQ GARS (Supplemental Figure 2). In sum, $\triangle$ ETAQ showed a severe defect in aminoacylation activity and, at best, a slight aberrant interaction with NRP1.

To definitively validate the pathogenicity of $\triangle E T A Q G A R S$ in vivo, we engineered a mouse model in which the mutation was introduced into the mouse Gars gene (Gars ${ }^{\triangle E T A Q /+}$ ) (see Methods). For subsequent preclinical studies, Gars ${ }^{\triangle E T A Q /+}$ mice were crossed to $\operatorname{Gars}^{h u E x x / h u E x x}$, a second mouse model engineered to harbor a "humanized" WT GARS exon 8 replacement in the mouse gene. The 50 amino acids encoded by exon 8 are $100 \%$ identical between mouse and human, although there are silent singlenucleotide differences between the mouse and human Gars/GARS exon 8 that could affect allele specificity of gene silencing. Our attempts to introduce these additional human changes when engineering the $\triangle \mathrm{ETAQ}$ allele were unsuccessful, and only the 12-bp deletion was incorporated.

This breeding produced a cohort of Gars ${ }^{\triangle E T A Q} / h u E x 8$ mice with Gars $^{h u E x x /+}$ littermate controls. Reverse transcriptase PCR using cDNA isolated from sciatic nerve of heterozygous mice revealed coexpression of $\triangle$ ETAQ and WT Gars (Supplemental Figure 3A). A Western blot analysis of brain homogenates using a polyclonal anti-GARS antibody confirmed that $\triangle$ ETAQ Gars did not alter GARS protein levels, suggesting that a stable transcript and protein products are produced from the $\triangle \mathrm{ETAQ}$ allele, as in patient fibroblasts (Supplemental Figure 3B).

At 12 weeks of age, Gars ${ }^{4 E T A Q / h u E x 8}$ and Gars $^{\text {huEx8/+ }}$ littermates were evaluated for features of primary neuropathy, as observed in other mouse models of CMT2D $(22,26)$. Gars $^{\triangle E T A Q / h u E x 8}$ mice displayed overt neuromuscular dysfunction and a reduction in 
A

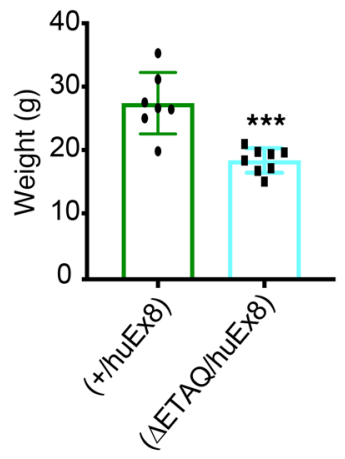

E
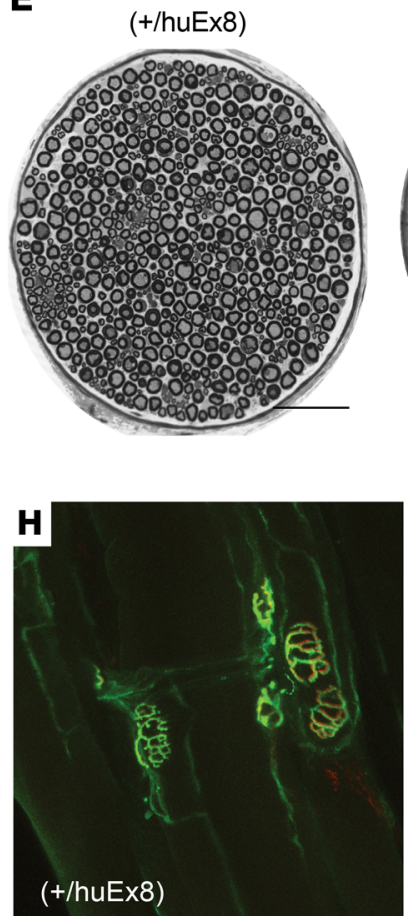

B
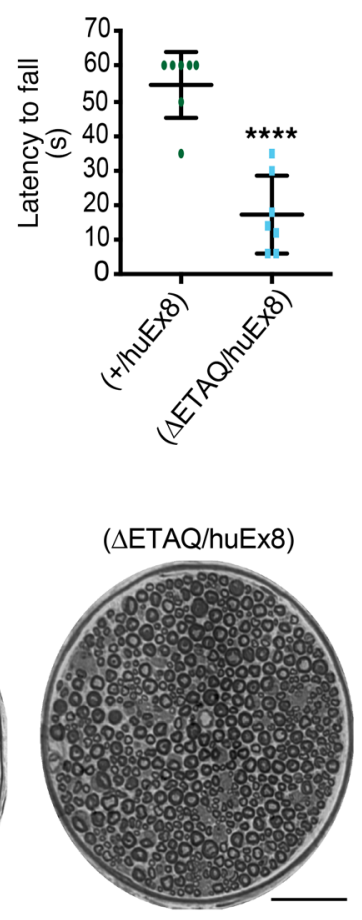

$\mathbf{F}$
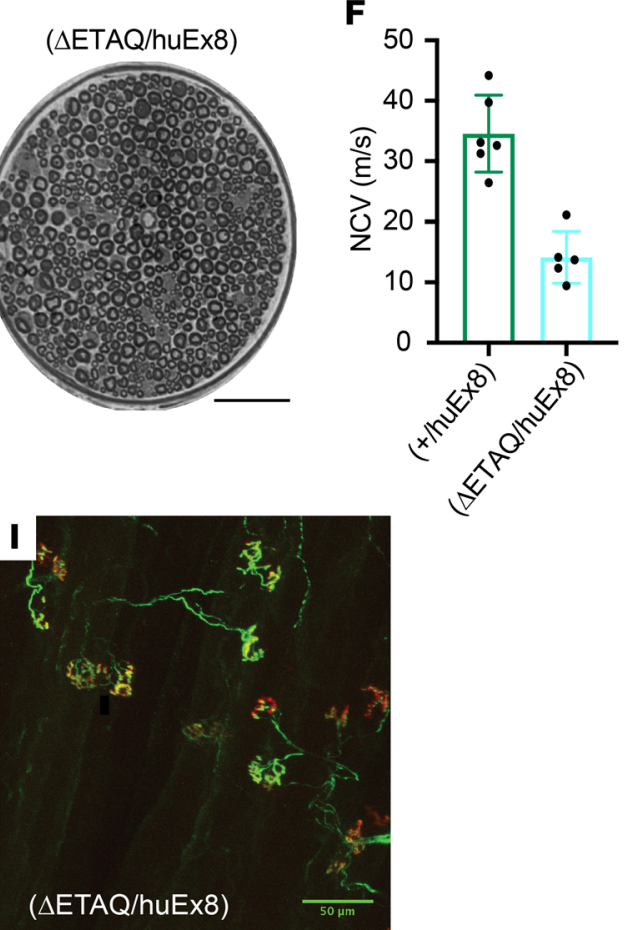

C

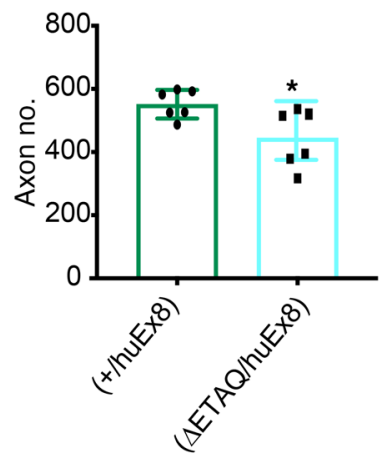

D

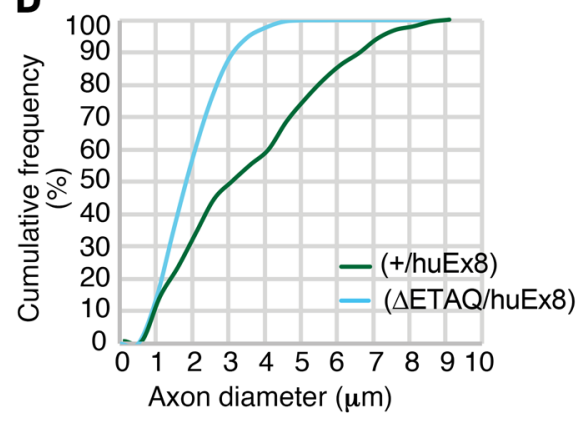

G Denervation

Partial innervation

- Full innervation
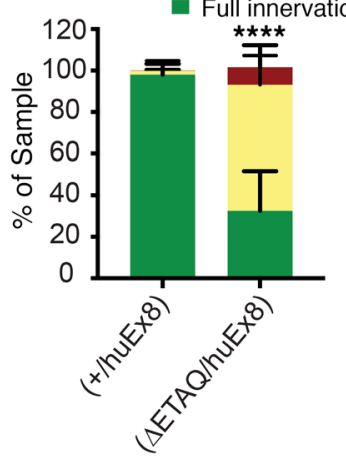

Figure 2. In vivo characterization of the $\triangle$ ETAQ GARS variant. (A) Gars ${ }^{4 E T A Q / h u E \times 8}$ mice and littermate controls were weighed at 12 weeks. Gars ${ }^{4 E T A Q / h u E \times 8}$ mice were significantly lighter, weighing $19 \pm 1.9 \mathrm{~g}(P=0.0006, n=8)$, compared with Gars ${ }^{\text {huEx } 8 /+}$ controls, which weighed $27.4 \pm 4.84 \mathrm{~g}(n=7)$. (B) Gross motor performance in Gars ${ }^{\triangle E T A O / h u E \times 8}$ mice was quantified using a wire hang test. While Gars ${ }^{\text {huEx } \times 8 /+}$ mice averaged $55 \pm 9.57$ seconds before letting go, Gars ${ }^{\triangle E T A Q / h u E \times 8}$ mice $(n=8)$ fell after only $17.3 \pm 11.3$ seconds. (C) Myelinated axon number in the motor branch of the femoral nerve was reduced by $21 \%$ from $551 \pm 45$ axons in littermate controls to $438 \pm 92$ axons in Gars ${ }^{A E T A O Q / h u E \times 8}$ ( $n=6$ mice per genotype). (D) Axon diameters were reduced, as shown in a cumulative histogram ( $P$ $<0.0001$, Kolmogorov-Smirnov test, average diameter $1.6 \pm 0.8 \mu \mathrm{m}, n=6$ ), in comparison with Gars ${ }^{+/ h u E x 8}$ littermates $(3.3 \pm 2.198 \mu \mathrm{m}, n=6)$. (E) Representative images of femoral motor nerve cross sections. (F) Nerve conduction velocity (NCV) was reduced from $35 \pm 6.29 \mathrm{~m} / \mathrm{s}$ in littermate controls to $13.5 \pm 4.1$ $\mathrm{m} / \mathrm{s}$ in Gars ${ }^{4 E T A Q / h u E \times 8}$ mice $\left(P=0.0002, n=6\right.$ Gars $^{4 E T A Q / h u E \times 8}, n=7$ Gars $\left.^{\text {huE } \times 8 /+}\right)$. (G) Neuromuscular junctions (NMJs) from the plantaris muscle showed partial innervation and denervation, scored based on the overlap between pre- and postsynaptic staining. (H and I) Representative images of NMJ morphology and innervation are shown. Differences in body weight, grip strength, conduction, and axon number between genotypes were statistically evaluated using a 2-way Student's $t$ test; axon diameter was evaluated by a Kolmogorov-Smirnov test. Significant difference in overall percentage NMJ innervation was determined by 2-way ANOVA with Tukey's honestly significant difference (HSD) post hoc comparisons. For all analyses, ${ }^{*} P<0.05,{ }^{* * *} P<0.001,{ }^{* * * *} P<0.0001$ represent post hoc significance between genotypes. Values are mean \pm SD. Scale bars: $100 \mu \mathrm{m}(\mathbf{E}) ; 50 \mu \mathrm{m}(\mathbf{H}, \mathbf{I})$.

body weight $(P=0.0006)$ and grip strength $(P=0.0002)$ compared with Gars ${ }^{h u E x 8 /+}$ controls (Figure 2, A and B). Histological changes in Gars $^{\triangle E T A Q / h U E x 8}$ mice were observed in cross sections of the motor branch of the femoral nerve, including an overall decrease in axon number $(P=0.0293)$ and axon diameter $(P<0.0001)$ (Figure 2 , $\mathrm{C}-\mathrm{E})$. The axon diameters were reduced in the sensory branch of the femoral nerve, but the number of myelinated axons was unchanged (Supplemental Figure 4, A and B). Nerve conduction velocities (NCVs) were reduced by $62 \%$, falling from $35 \pm 6.29$ $\mathrm{m} / \mathrm{s}$ in control animals to $13.5 \pm 4.1 \mathrm{~m} / \mathrm{s}(P=0.0002)$ in the sciatic nerve in mutant mice (Figure $2 \mathrm{~F}$ ). This decrease was consistent with other mouse Gars neuropathy models and with some patients with GARS-mediated peripheral neuropathy (CMT2D). There was a concomitant disruption of neuromuscular junctions (NMJs) in distal muscles. While postsynaptic receptor fields of NMJs in the plantaris muscle were fully occupied by motor nerve terminals 
A

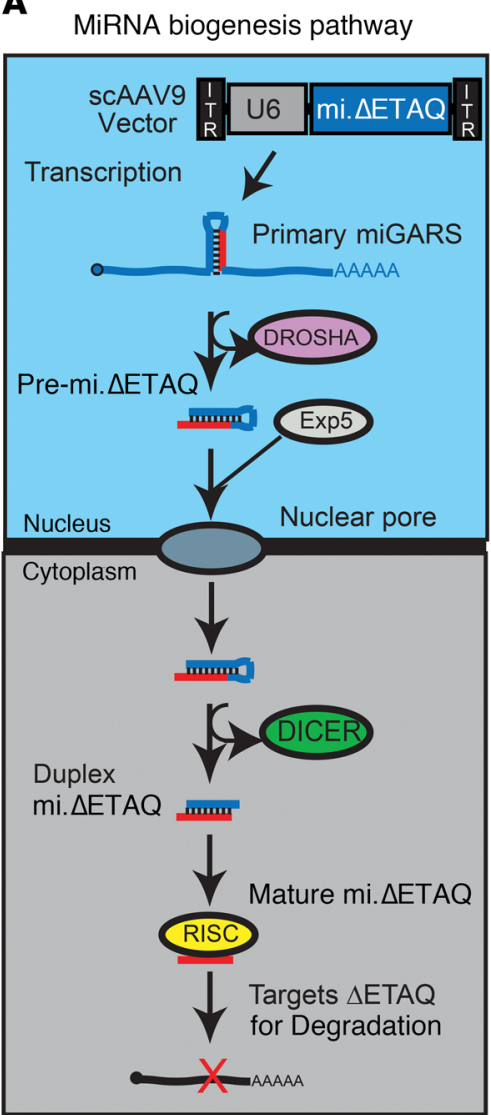

B
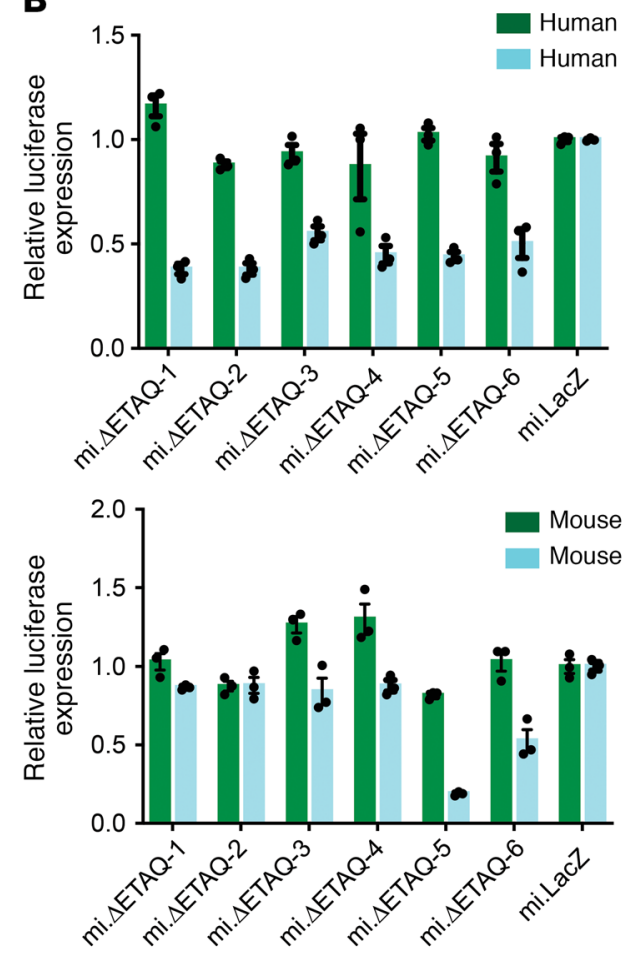

C

WT AUGCCUGGGUACUUGAGACCAGAAACUGCACAGGGACU

|| ||||||||||||||||

ACCCAUGGACUCUGGCCCUUAA mi. $\triangle E T A Q$

||||||||||||||||||||||||

$\triangle E T A Q$ AUGCCUGGGUACUUGAGACCGGGGAUUUUCUUGAAUUUC

M P G Y L R P G I F L N F

Figure 3. Generation of $\triangle E T A Q$-targeting miRNA shuttles. (A) Therapeutic miCARS miRNAs utilize naturally occurring RNAi biogenesis and gene silencing pathways in targeting cells. Each miGARS or control sequence was cloned as a DNA template downstream of a U6 promoter and then delivered to cells via plasmid transfection (in vitro) or within scAAV9 particles in vivo (depicted here). Once in the target cell nucleus, primary miRNA constructs are transcribed and then processed by the RNAses DROSHA and DICER and the nuclear export factor exportin-5 (Exp5). The mature antisense strand (red line) incorporates into the RNA-induced silencing complex (RISC) to elicit sequence-specific degradation of the mutant Gars mRNA. (B) MiRNAs were tested in vitro by cotransfection of HEK293 cells with U6-miGARS, or control, plasmid miRNA and a dual-luciferase reporter plasmid containing 1 of 4 target genes cloned into the 3'-UTR of Renilla luciferase: WT human CARS, human $\triangle \mathrm{ETAQ}$ GARS, WT mouse Gars, or the mouse Gars gene containing the same ETAQ deletion. Target gene silencing was then determined by measurement of the ratio of Renilla to firefly luciferase. The values are reported as mean ratios \pm SEM. (C) The sequence of the guide strand of the lead mi. $\triangle E T A Q$ and its complementarity to both the WT and $\triangle \mathrm{ETAQ}$ GARS gene. The 4-amino acid deletion is shown in red. Base pairing between the miRNA and target genes is shown with vertical lines, with red lines indicating wobble $\mathrm{G}-\mathrm{U}$ bonds present in RNA duplexes.

in control littermates, $60 \% \pm 14.2 \%$ of NMJs were partially occupied and $8.5 \% \pm 9.9 \%$ were completely denervated in $\triangle \mathrm{ETAQ}$ mice (Figure 2, G-I). Defects in NMJ innervation were evident at 6 weeks of age, and examples of fully innervated, partially innervated, and denervated junctions are shown in Supplemental Figure $4, C-G$, and resemble those in previously reported Gars mice (22, 26-28). Thus, Gars ${ }^{A E T A / / h u E x B}$ mice display primary features of peripheral neuropathy similar to other established mouse models of CMT2D, confirming that the $\triangle$ ETAQ GARS mutation is pathogenic $(22,26,29,30)$.

To test whether allele-specific knockdown of mutant GARS using RNAi would be efficacious, we first engineered a miRNA shuttle designed to specifically target $\triangle$ ETAQ transcripts for degradation (Figure 3, A and B, and Supplemental Figure 5). We designed 6 different mir-30-based artificial miRNA shuttles (mi. $\triangle$ ETAQ1-6) with a mature guide strand designed to specifically target both human and mouse mutant Gars $\triangle \mathrm{ETAQ}$ mRNA for degradation (Figure 3B and Supplemental Figure 5). To identify a lead miRNA shuttle, we cloned each mi. $\triangle E T A Q$ sequence into a U6 promoter-driven expression cassette. These were used in an initial in vitro dual-luciferase screening assay (31), in which we cloned the $\triangle \mathrm{ETAQ}$ or WT Gars target sequences into the 3'-UTR of sea pansy (Renilla reniformis) luciferase and used firefly luciferase as a standard. Knockdown efficiency and disease allele specificity were tested following cotransfection of the reporter plasmid and each U6.mi. $\triangle$ ETAQ shuttle (or a control vector) into HEK293 cells. The most effective construct (mi. $\triangle \mathrm{ETAQ} 5$, now referred to as mi. $\triangle \mathrm{ETAQ}$ ) (Figure 3, B and C) was cloned into a self-complementary adeno-associated viral vector (serotype 9; scAAV9) for in vivo delivery (Figure 3B and Supplemental Figure 5).

To establish the proof of principle of this approach in vivo, we tested whether the reduction of mutant Gars expression before disease onset could prevent the neuropathy in Gars ${ }^{\triangle E T A Q / h u E x B}$ mice. A dose of approximately $2.6 \times 10^{11}$ viral genomes (vg) of scAAV9.mi. $\triangle \mathrm{ETAQ}$ or scAAV9.mi.LacZ (expressing a control miRNA targeting the $E$. coli LacZ gene) was delivered with an intracerebroventricular (i.c.v.) injection at postnatal day 0-1 (PO-P1) to Gars ${ }^{\triangle E T A Q / h u E x s}$ and littermate control (Gars $\left.{ }^{h u E x s / h u E x s}\right)$ pups. All mice were evaluated for established signs of neuropathy at 4 weeks of age, approximately 1.5 weeks after the initial onset of overt signs of neuropathy. Gars ${ }^{\triangle E T A Q / h u E x 8}$ mice treated with scAAV9.mi. $\triangle$ ETAQ showed improvement in a wire hang test of grip strength, increased muscle-to-body-weight (MW/BW) ratios, and improved sciatic nerve conduction velocity (NCV) compared with control-treated $\triangle$ ETAQ mice (Figure 4, A-C). Examination of cross sections of the motor branch of the femoral nerve revealed that scAAV9. mi. $\triangle E T A Q$ treatment prevented the axon loss and lessened the decrease in axon diameters observed in untreated and scAAV9. 
A

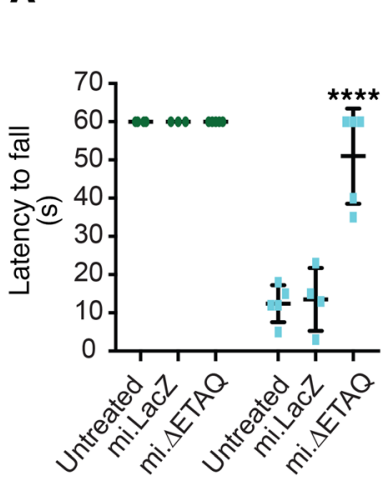

B

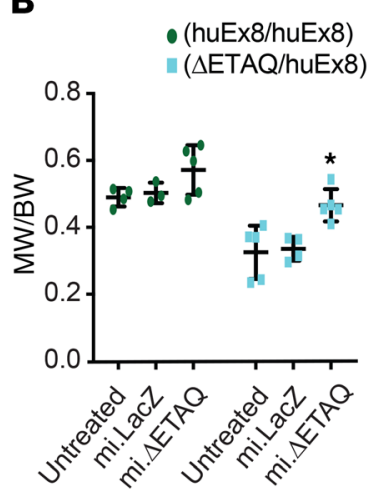

C

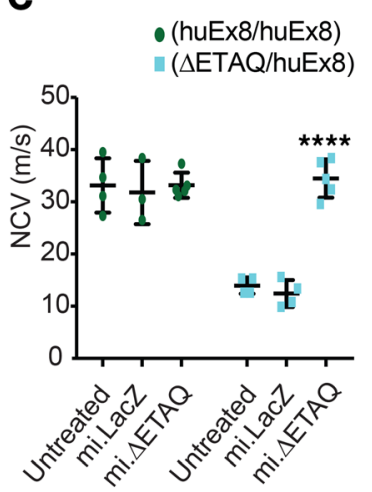

D

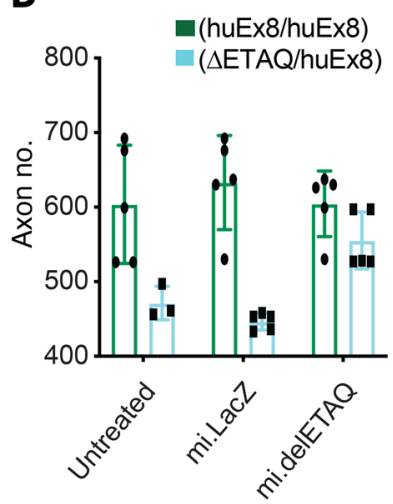

E

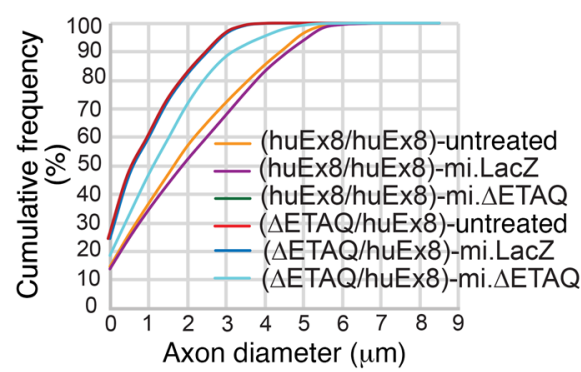

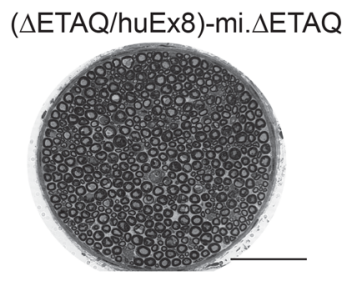

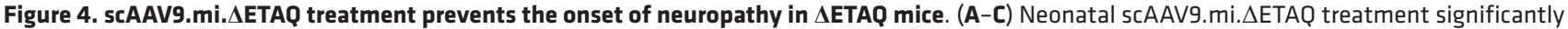
prevented deficits in gross motor performance quantified by the wire hang test $(P=0.0001)$ as well as reductions in MW/BW ratios $(P=0.0315)$ and NCVs $(<0.0001)$, in comparison with untreated or vehicle-treated Gars ${ }^{4 E T A O / h u E x 8}$ mice. (D-F) Quantification of axon number and axon size indicated that scAAV9. mi. $\triangle$ ETAQ could partially prevent axon loss $(P=0.0272)$ and reductions in axon diameter $(P \leq 0.0001)$ in comparison with scAAV9.mi.LacZ-treated $\triangle$ ETAQ mice, as shown in cross sections of the motor branch of the femoral nerve. Axon diameter was analyzed using a Kolmogorov-Smirnov normality test, while all other outcome measures were analyzed using a 2-way ANOVA with Tukey's HSD post hoc comparisons. ${ }^{*} P<0.05$, ${ }^{* * * *} P<0.0001$ represent post hoc

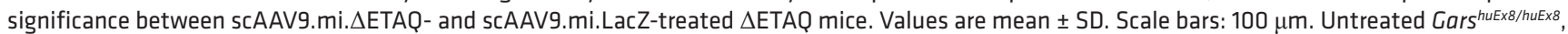

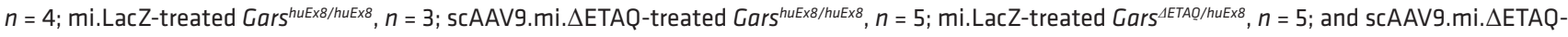
treated Gars ${ }^{A E T A O / h u E x 8}, n=5$.

mi.LacZ-treated $\triangle$ ETAQ mice (Figure 4, D-F). Injection with scAAV9.mi. $\triangle$ ETAQ (or scAAV9.mi.LacZ) did not cause adverse effects in control mice. Collectively, these data provide the proof of concept that allele-specific knockdown using virally delivered RNAi may be an effective approach for treating CMT2D.

We next delivered scAAV9.mi. $\triangle$ ETAQ to cohorts of both earlysymptomatic (5-week-old) and late-symptomatic (9-week-old) Gars $^{\triangle E T A Q / h u E x 8}$ mice and littermate controls via a single intrathecal injection into the lumbar spinal cord. When left untreated, 5-week-old early-symptomatic Gars ${ }^{\triangle E T A Q / h u E x 8}$ mice undergo active axon loss, while axon loss slows and muscle atrophy accelerates in 9-week-old late-symptomatic $\triangle \mathrm{ETAQ}$ mice.

The scAAV9.mi. $\triangle$ ETAQ-treated early-symptomatic Gars ${ }^{4 \mathrm{ETAQ} / \mathrm{huEx}}$ mice displayed enhanced grip strength and increases in body weight starting at about 5 weeks after treatment compared with untreated controls (Figure 5A). When analyzed for primary signs of neuropathy at 7 weeks after treatment, early-symptomatic Gars $^{4 E T A Q / h u E x}$ mice also exhibited significant increases in MW/BW ratios, NCV, and NMJ innervation and a reduction in axon loss but no improvement in axon size compared with untreated Gars $^{\triangle E T A Q / h u E x 8}$ mice (Figure 5, B-D and F). When treated with scAAV9.mi.AETAQ at 9 weeks of age, Gars ${ }^{\triangle E T A Q / h u E x 8}$ mice gained weight and displayed enhanced grip strength starting at 5-7 weeks after treatment (Figure $5 \mathrm{~A})$. While $\mathrm{MW} / \mathrm{BW}$ ratios were not improved and axon loss and atrophy were not prevented, scAAV9.mi. $\triangle$ ETAQ did improve NCV and NMJ innervation (Figure 5, B, E, and F). Analysis of mRNA from sensory dorsal root ganglia (DRGs), which are also transduced by scAAV9, via pyrosequencing indicated that mutant Gars mRNA levels were significantly reduced in scAAV9.mi. $\mathrm{ETAQ}$-treated mice (Supplemental Table 2).

We also evaluated possible off-target effects of the RNAi. In addition to prediction programs, we also empirically tested for gene expression changes and found 325 genes that were altered in their expression with an FDR less than 0.05 based on RNASeq of patient fibroblasts transfected with the mi. $\triangle$ ETAQ construct in comparison with eGFP transfection as a control. The physiological significance of these changes is unknown, and the transcriptome of fibroblasts will be only partially shared with that of neurons. To evaluate possible adverse effects in vivo, WT mice were treated with the same dose of vector by intrathecal injection (30 seven-week-old female mice comprising 3 groups: 10 mi. $\triangle$ ETAQ treated, $10 \mathrm{mi} . \mathrm{LacZ}$ treated, and 10 untreated). No adverse effects other than a mild increase in germinal center activity in the spleen were seen 10 weeks after treatment after full necropsy and evaluation by a veterinary pathologist blinded to treatment.

We confirmed the efficacy of allele-specific knockdown in a second mouse model of CMT2D, $\operatorname{Gars}^{P 278 K Y /+}$ (22). A miRNA 
A

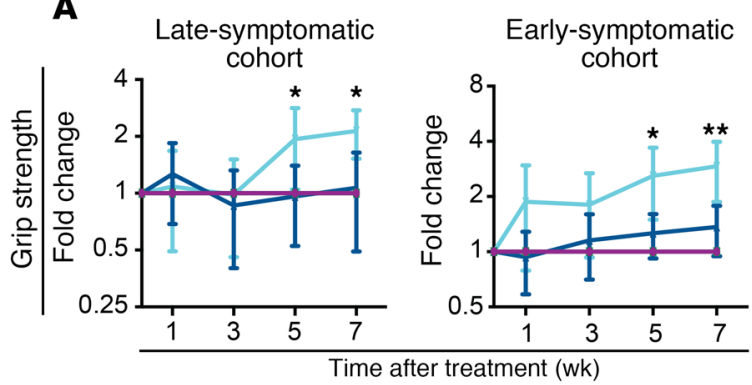

Late-symptomatic cohort

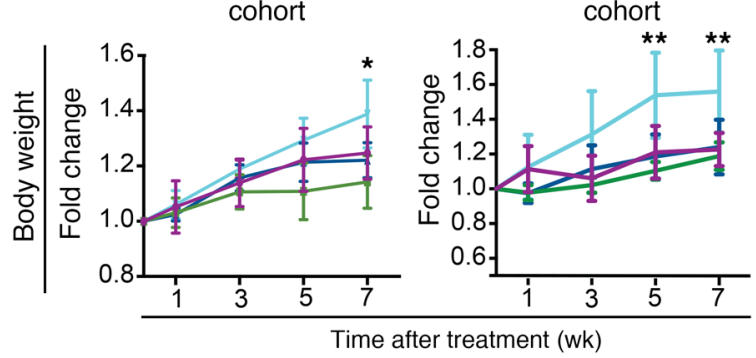

(huEx8/huEx8)-mi.LacZ (huEx8/huEx8)-mi. $\triangle E T A Q$

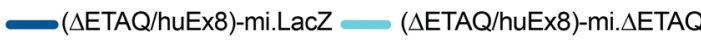

B
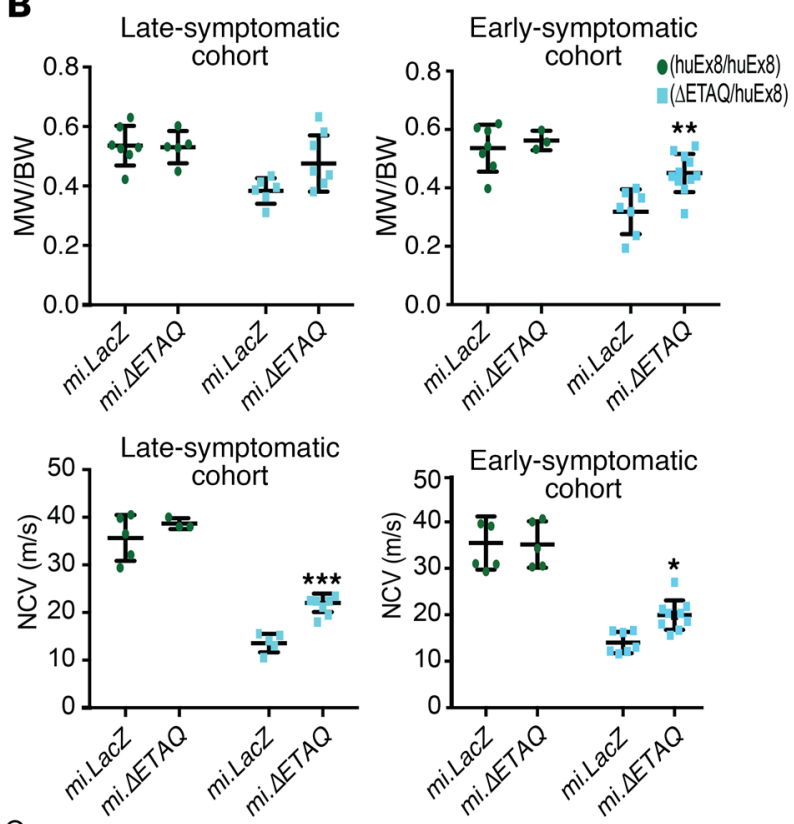

C

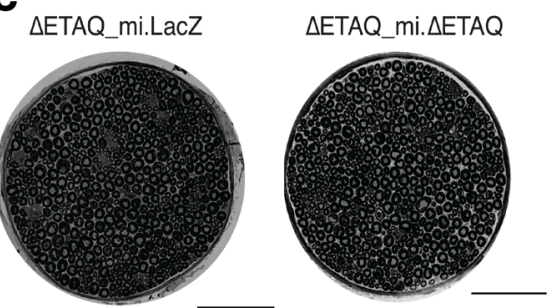

D

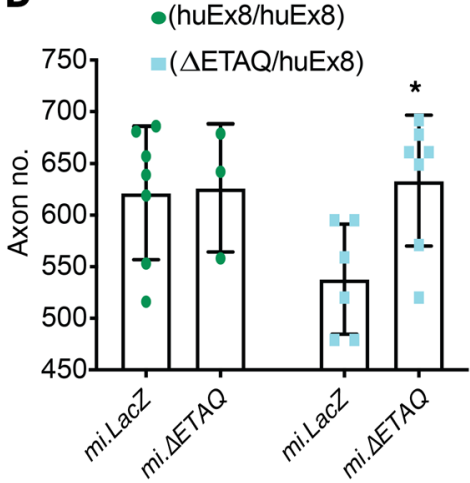

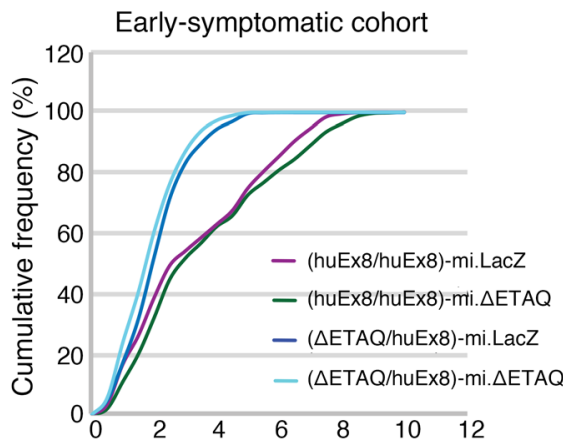

E

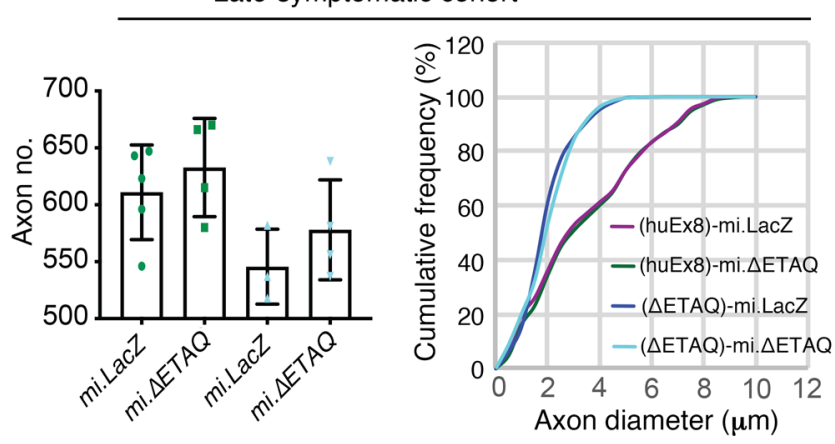

$\mathbf{F}$

Early-symptomatic cohort
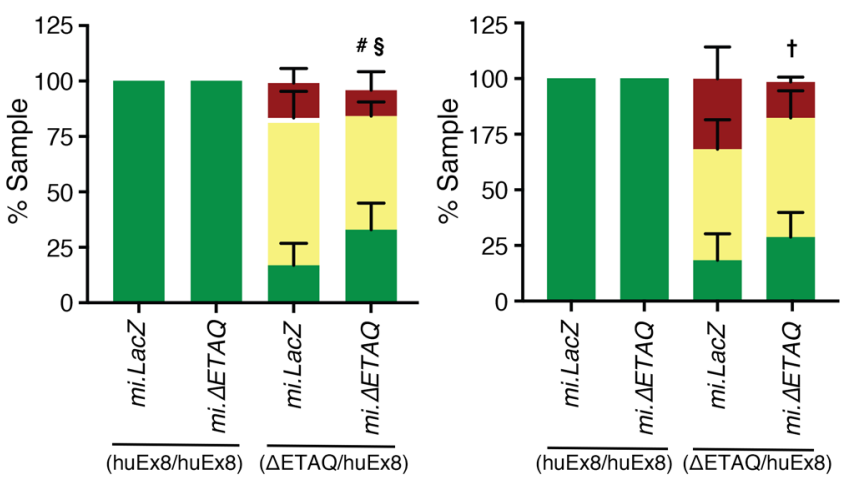
Figure 5. Post-onset therapeutic effects of scAAV9.mi.AETAQ. (A) Reduction in mutant Gars expression improved grip strength and increased body weight in early- and late-symptomatic Gars ${ }^{\triangle E T A Q} / h u E \times 8$ mice. Mi. $\triangle$ ETAQ-treated early- and late-symptomatic Gars ${ }^{\triangle E T A O / h u E x 8}$ mice exhibited enhanced grip strength and significant increases in body weight starting at approximately 5 weeks after treatment. When evaluated at 7 weeks after treatment for signs of neuropathy, these data correlate with trending increases in MW/ $\mathrm{BW}$ ratios and significant improvements in NCV in mi. $\triangle \mathrm{ETAQ}$-treated late-symptomatic Gars ${ }^{A E T A Q / h u E \times 8}$ mice. (B-D) Early-symptomatic Gars ${ }^{\triangle E T A Q / h u E \times 8}$ mice treated with mi. $\triangle E T A Q$ displayed significantly higher MW/BW ratios and faster NCVs (B), most likely resulting from the greater number of motor axons observed in cross sections of the motor branch of the femoral nerve, although improvement in axon diameter was not observed (C and D). (E) Prevention of axon loss was not observed in mi. $\triangle$ ETAQ-treated latesymptomatic Gars ${ }^{\triangle E T A O / h u E \times 8}$ mice. (F) Both early- and late- symptomatic overall displayed significant increases in NMJ innervation. Data were analyzed using a 1-way ANOVA followed by Tukey's HSD post hoc comparisons. Significant changes in axon diameter (E) were determined with a KolmogorovSmirnov test. ${ }^{*} P<0.05,{ }^{* *} P<0.01,{ }^{* *} P<0.001$ represent post hoc significance between mi.LacZ-treated and scAAV9.mi. $\triangle$ ETAQ-treated Gars ${ }^{\Delta E T A Q / h u E x 8}$ mice. "Significant difference in fully innervated NMJs; ${ }^{\S}$ significant difference in partially innervated NMJs; ‘'significant difference in denervated NMJs. Late-symptomatic cohort: mi.LacZ-treated Gars ${ }^{\text {huEx8/huEx }}, n=5-7$; scAAV9. mi. $\triangle$ ETAQ-treated Gars ${ }^{\text {huE } \times 8 / h u E \times 8}, n=3-5$; mi.LacZ-treated Gars ${ }^{\Delta E T A O / h u E \times 8}$, $n=6$; and scAAV9.mi. $\triangle$ ETAQ-treated Gars ${ }^{\triangle E T A Q / h u E \times 8}, n=7$. Early-symptomatic cohort: Gars ${ }^{\text {huEx8/huEx }}, n=6$ 6-7; scAAV9.mi. $\triangle$ ETAQ-treated Gars ${ }^{\text {huEx8/huEx8 }}$, $n=3-5$; mi.LacZ-treated Gars ${ }^{4 E T A Q / h u E \times 8}, n=7$; and scAAV9.mi. $\triangle$ ETAQ-treated Gars $^{\text {AETAQ/huEX8 }}, n=9-11$. Values are mean \pm SD. Scale bars: $100 \mu \mathrm{m}$.

shuttle targeting the mouse P278KY allele was optimized as before and packaged into scAAV9 (Supplemental Figure 6). As with the $\triangle \mathrm{ETAQ}$ vector, improvements were observed in neonatal, earlyand late-symptomatic Gars ${ }^{P 28 K Y /+}$ mice that were treated with 1 $\times 10^{11} \mathrm{vg}$ of scAAV9.mi.P278KY delivered by i.c.v. (neonates) or intrathecal (adults) injection (Figure 6 and Supplemental Figure 7). $\operatorname{Gars}^{\mathrm{P} 278 K \mathrm{Y} /+}$ mice treated with the therapeutic vector at $\mathrm{PO}-\mathrm{P} 1$ showed WT performance in the wire hang test (Figure 6A), and did not show signs of muscle atrophy, reduced NCV, or axon loss when examined at 4 weeks of age (Figure 6, B-D). Axon diameters in the motor branch of the femoral nerve and innervation at the NMJ were both improved over untreated or scAAV9.mi.LacZtreated mice, but were not preserved to WT levels (Figure 6, E-H). No adverse effects were noted in WT mice treated with this vector. When treated after the onset of disease at 5 or 9 weeks of age, these mice improved in their grip strength and body weights over the next 7 weeks to a greater extent than mice treated with the lac $Z$ vector (Supplemental Figure 7). While muscle atrophy was reduced with treatment at 5 weeks of age, it was not improved with treatment at 9 weeks, and NCV was not improved at either time point (Supplemental Figure 7). However, innervation at the NMJ was improved at both ages (Supplemental Figure 7).

The therapeutic effects of scAAV9.mi.P278KY were dose dependent, and were greater with i.c.v. delivery compared with a systemic, i.v. injection delivering the same total dose (Supplemental Figure 8). With i.c.v. delivery, doses including $1 \times 10^{10}, 5 \times$ $10^{10}$, and $1 \times 10^{11} \mathrm{vg}$ all showed dose-dependent improvement over untreated mice, with $1 \times 10^{11}$ achieving near-complete prevention of the neuropathy (Figure 6 and Supplemental Figure 8). Delivering $1 \times 10^{11} \mathrm{vg}$ systemically by i.v. injection produced benefit comparable to $1 \times 10^{10} \mathrm{vg}$ delivered directly to the nervous system by i.c.v. injection (Supplemental Figure 8), consistent with the need to target the nervous system and not peripheral tissues.

The beneficial effects of i.c.v. delivery of the high dose $(1 \times$ $10^{11} \mathrm{vg}$ ) of scAAV9.mi.P278KY at PO lasted at least 1 year (Figure 7). Treated mice maintained more normal body weights than sham-treated controls (Figure 7A). They also maintained their normal performance in the wire hang test (Figure 7B). One year after treatment, the Gars ${ }^{\text {P27RKY/+ }}$ mice did not show signs of muscle atrophy, reduced NCV, axon loss, or axon atrophy (Figure 7, C-G). Thus, consistent with the perdurance of AAV9, a single dose at birth prevented neuropathy for at least 1 year.

The outcomes in post-onset studies in both genotypes were variable, likely because of the challenges in intrathecal delivery in mice. However, the knockdown efficacy of mutant Gars transcripts within DRGs (Supplemental Tables 2-4) correlated with therapeutic outcomes within both post-onset studies (Supplemental Figure 9, A, B, E, and F). This correlation was stronger with mRNA isolated from DRGs than when outcomes were compared with mutant Gars mRNA levels in liver, another tissue transduced by scAAV9 (Supplemental Figure 9, C, D, G, and H). This is consistent with the benefit coming from the transduction of cells with direct delivery to the nervous system and not from transduction of peripheral organs.

\section{Discussion}

In this study we examined a new de novo variant of GARS, $\triangle E T A Q$, identified in a patient with an early-onset motor neuropathy. Although not unprecedented, the severity and onset of this case are atypical for CMT2D (14). Biochemical characterization of the mutation revealed a loss of activity in the charging of glycine onto tRNA ${ }^{\text {Gly }}$, consistent with other pathogenic alleles of GARS (18). However, in contrast to other pathogenic alleles (23), the mutant protein does not strongly bind to NRP1. Given the atypical clinical presentation and biochemical properties of the $\triangle$ ETAQ variant, we confirmed its pathogenicity by introducing the same change into the mouse Gars gene. Mice carrying this dominant mutation developed a severe, early-onset peripheral neuropathy, confirming that it is indeed disease-causing. We next developed an allele-specific-knockdown vector to target mutant Gars transcripts while leaving the WT Gars mRNA intact. RNAi sequences were optimized in vitro and cloned into a miR30 shuttle construct driven by a U6 promoter, which was packaged into scAAV9 for in vivo delivery. Treatment of the mouse model at birth by i.c.v. injection was able to largely prevent the onset of neuropathy, and delivery by intrathecal injection after the onset of symptoms still had benefit, though the degree of benefit decreased as delivery of the treatment was delayed. These results were confirmed in a second mouse model of CMT2D, carrying a mutation (P278KY) not found in patients. In these mice, an allele-specific knockdown vector delivered as above produced similar benefit, both before and after onset. These effects were dose dependent and persisted for at least 1 year. The improvement in neuropathy phenotypes correlated with the degree of knockdown of the mutant Gars mRNA. Thus, our findings support the feasibility of virally delivered RNAi for allele-specific knockdown as a treatment strategy for a dominant neuropathy caused by mutations in an essential gene. 
A

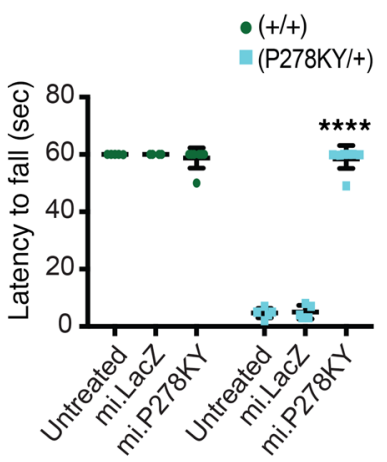

B

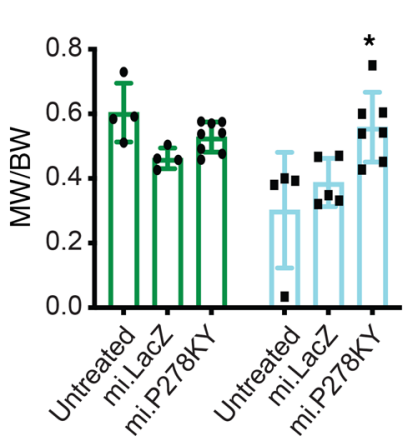

C

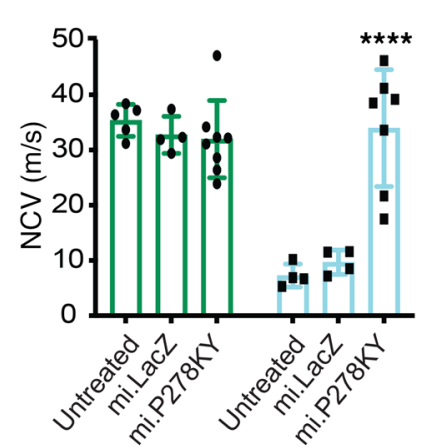

D

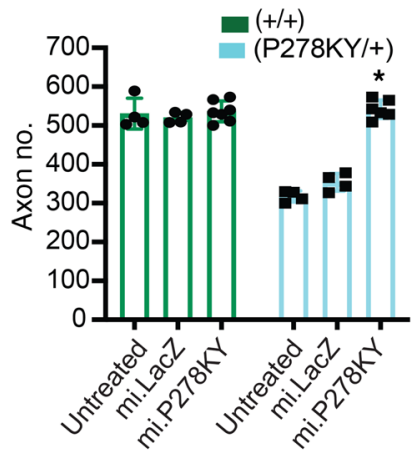

E

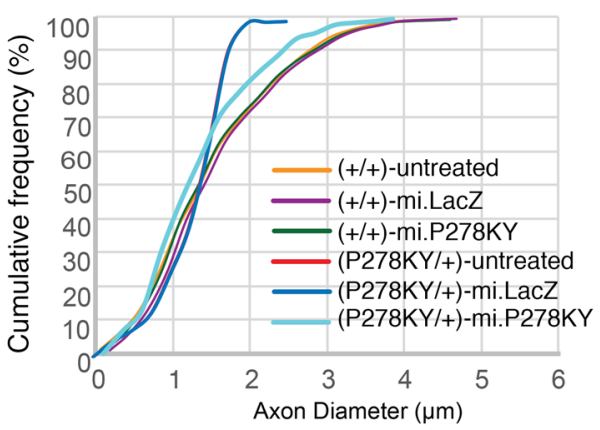

F

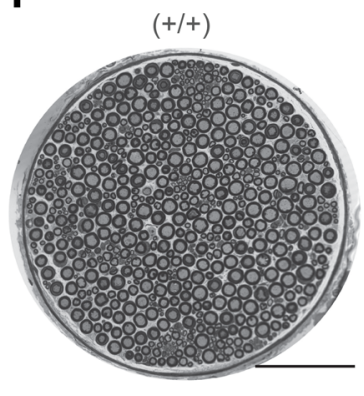

(P278KY/+)-mi.P278KY

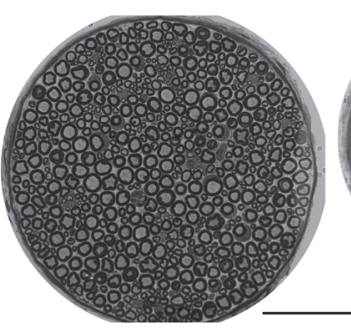

(P278KY/+)
G

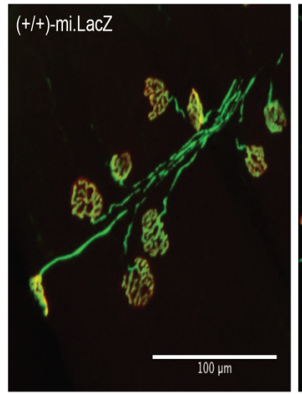

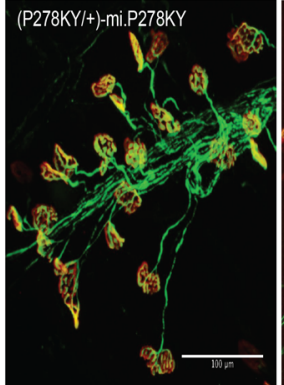

H

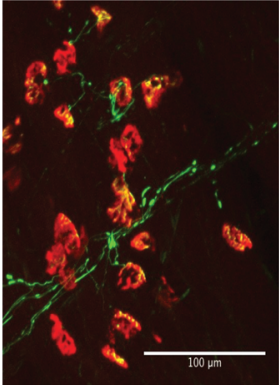

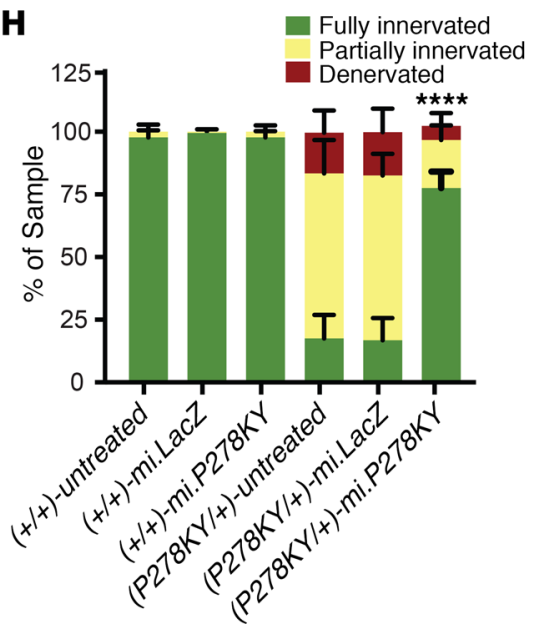

Figure 6. Reduction of mutant Gars by RNAi prevents neuropathy in Gars ${ }^{\text {P278KY/+ }}$ mice. (A and B) Neonatal scAAV9.mi.P278KY treatment prevented deficits in gross motor performance quantified at 4 weeks of age by the wire hang test $(P=0.0001)(\mathbf{A})$ and reductions in MW/BW ratios $(P=0.0463)(\mathbf{B})$ in comparison with untreated and vehicle-treated P278KY mice. (C) NCVs were also significantly improved $(P \leq 0.0001)$ in treated P278KY mice. (D) Quantification of axon number within cross sections of the motor branch of the femoral nerve showed that while axon number was reduced by $17 \%$ in control-treated P278KY mice, axon counts in scAAV9.mi.P278KY-treated P278KY mice ( $589 \pm 15$ axons) were similar to those in untreated control littermates (600 \pm 11 axons). (E) scAAV9. mi.P278KY treatment also restored the presence of large-diameter axons; average axon diameter was $1.98 \pm 4.47 \mu \mathrm{m}$ in control-treated P278KY mice, $2.71 \pm$ $3.71 \mu \mathrm{m}$ in scAAV9.mi.P278KY-treated P278KY mice, and $3.84 \pm 3.74 \mu \mathrm{m}$ in untreated Gars ${ }^{+/+}$mice. (F) Representative images of cross sections of the motor branch of the femoral nerve isolated from untreated Gars ${ }^{+/+}$, Gars ${ }^{P 278 K Y /+}$, and scAAV9.mi.P278KY-treated Gars ${ }^{P 278 K Y /+}$ mice. (G) Representative images of NMJ morphology isolated from plantaris muscle. $(\mathbf{H})$ While over $70 \%$ of the NMJs were partially or completely denervated in control-treated Gars ${ }^{P 278 k V /+}$ mice by 4 weeks of age, less than $30 \%$ of NMJs showed any degree of denervation in scAAV9.mi.P278KY-treated Gars ${ }^{P 278 K Y /+}$ mice. Numbers for all outcome measures:

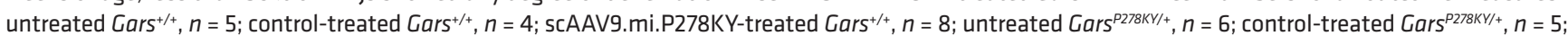
scAAV9.mi.P278KY-treated Gars ${ }^{\mathrm{P27} 8 \mathrm{KV} /{ }^{+}}, n=7$. Significance in $\mathbf{A}-\mathbf{D}$ and $\mathbf{H}$ was determined by 2-way ANOVA with Tukey's HSD post hoc comparisons. Significant changes in axon diameter (E) were determined with a Kolmogorov-Smirnov test. ${ }^{*} P<0.05$, ${ }^{* * *} P<0.0001$ represent post hoc significance between mi.LacZ-treated and scAAV9.mi.P278KY-treated Gars ${ }^{P 278 K Y /+}$ mice. Values are mean \pm SD. All scale bars: $100 \mu \mathrm{m}$. 
A
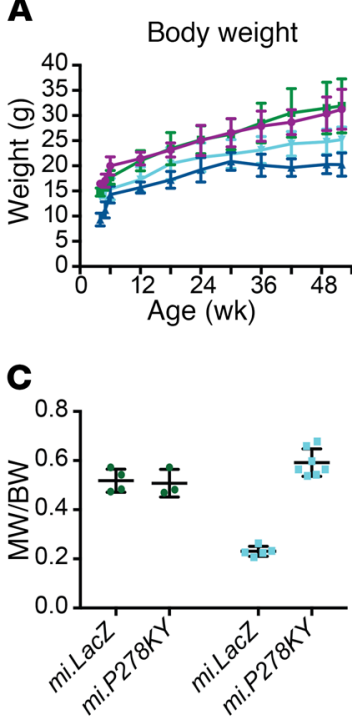

B

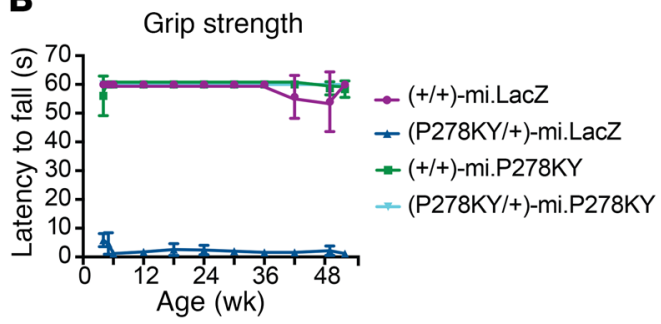

D

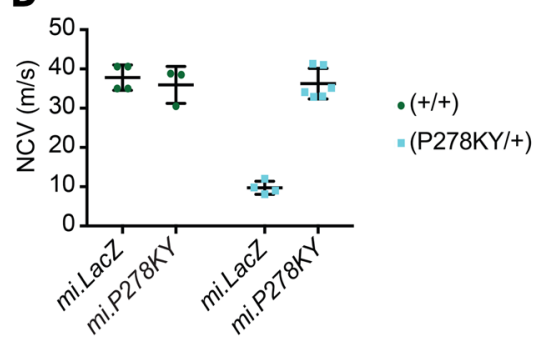

E

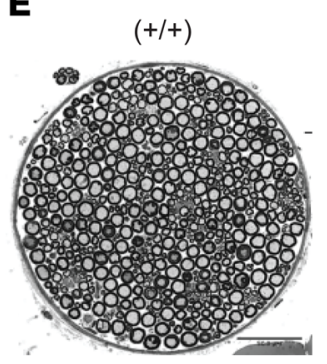

(P278KY/+)-mi.P278KY

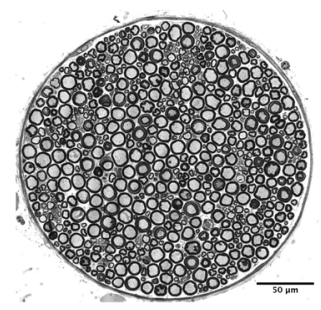

(P278KY/+)

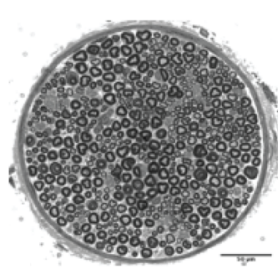

$\mathbf{F}$

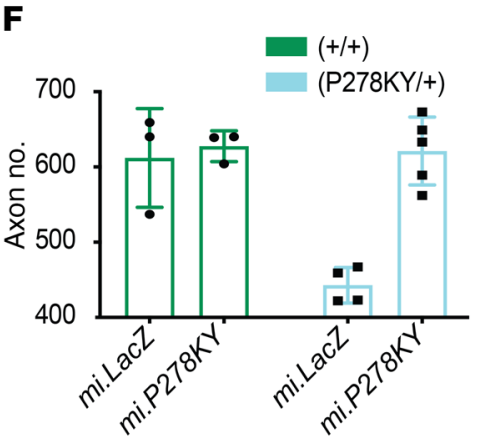

G

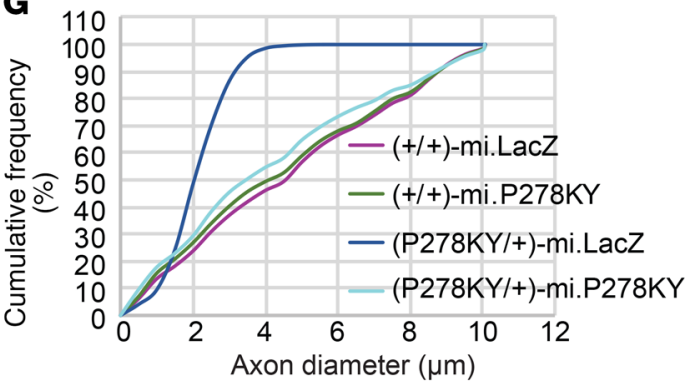

Figure 7. Long-term therapeutic effects of neonatal scAAV9.mi.P278KY treatment. (A and B) scAAV9.mi.P278KY-treated P278KY mice displayed increases in body weight (A) starting at 24 weeks after treatment and in grip strength (B) throughout the course of 1 year compared with vehicle control-treated P278KY mice. (C and $\mathbf{D}$ ) When evaluated for primary signs of neuropathy at 1 year after treatment, treated P278KY mice exhibited greater MW/BW ratios (C) and faster NCVs (D). (E-G) scAAV9.mi.P278KY treatment could preserve all populations of axons in the motor branch of the femoral nerve at 1 year after treatment. Significance in $\mathbf{A}$ and $\mathbf{B}$ was determined by 1-way ANOVA with Tukey's HSD post hoc comparisons. Significance in $\mathbf{C}, \mathbf{D}$, and $\mathbf{F}$ was determined by 2-way ANOVA with Tukey's HSD post hoc comparisons. Significant changes in axon diameter (G) were determined with a Kolmogorov-Smirnov test. Mi.LacZ-treated Gars+/+, $n=3$; scAAV9.mi.P278KY-treated Gars+/+, $n=3$; mi.LacZ-treated GarsP278 ${ }^{K Y /+}$, $n=5$; and scAAV9.mi.P278YK-treated $\operatorname{Cars}^{P 278 K Y /+}, n=7$. Values are mean \pm SD.
The disease mechanism through which mutant forms of GARS cause CMT2D is not clear. Variants of GARS that result in neuropathy impact the enzymatic activity of the protein to varying degrees. Since alleles that do not produce a mutant protein do not cause a dominant disease in mice or humans, a loss-of-function mechanism underlying the neuropathy would have to be through a dominant-negative mechanism. Alternatively, neomorphic gainof-function activities are consistent with findings in mouse and Drosophila models $(20,32)$. Mutant forms of GARS bind to NRP1, an important neurodevelopmental receptor that transduces both VEGF and semaphorin signals, whereas WT GARS does not bind NRP1 (23). This aberrant interaction interferes with VEGF binding, possibly constituting a pathogenic neomorphic activity of the mutant protein. However, our finding that the $\triangle \mathrm{ETAQ}$ form of GARS does not have a strong interaction with NRP1 indicates that while this mechanism may still contribute to the disease, it is not essential for pathogenicity. Importantly, our allele-specific knockdown approach applies equally well to a dominant-negative or a neomorphic mechanism.

Our results also serve as a preclinical demonstration of the feasibility of RNAi-mediated allele-specific knockdown as a therapeutic strategy. Pre-onset treatments were able to almost completely prevent the neuropathy. This provides strong proofof-concept data for the effectiveness of knocking down mutant GARS. Post-onset treatment still provided benefit, though this was less complete and diminished the longer treatment was withheld. The post-onset treatment may stop or slow the progression of axon loss. This is best demonstrated by the reduction in axon loss seen in $\triangle$ ETAQ mice treated at 5 weeks of age compared with untreated mutant mice (Figure 5D), but there was no similar reduction with treatment at 9 weeks of age (Figure $5 \mathrm{E}$ ), an age by which most of the axons have already degen- 
erated. Even with axon loss, some of the residual benefit may come from improved synaptic transmission at NMJs, consistent with the improved innervation and with previous findings that synaptic transmission is progressively impaired in Gars mutant mice (28).

Another important consideration for the translational potential of this approach is whether we can achieve sufficient allelic specificity with single-base pair mutations. The mutations that we targeted here differed from WT sequence by 5 (P278KY) or 12 ( $\triangle$ ETAQ) bp, making them relatively good targets for specific RNAi sequences. Allele specificity of RNAi for single-nucleotide differences will depend on surrounding local sequence, but is precedented, and efficiency can be improved by addition of additional mismatches to the RNAi sequence (33-35). Therefore, a similar allele-specific knockdown strategy may be effective for additional alleles of GARS. An alternative strategy that would apply equally to any allele of GARS would be to knock down all GARS transcripts, mutant and WT, using a nonspecific GARS RNAi, and, in the same vector, provide an RNAi-resistant WT cDNA. This strategy has been used for $\alpha_{1}$-antitrypsin (36). Other strategies, such as allele-specific antisense oligonucleotides or allele-specific CRISPR/Cas9 gene editing, could also be used to silence mutant GARS alleles while preserving WT GARS, but would require additional development.

The reduced efficacy of the treatment after the onset of neuropathy will be complicated to dissect. It is possible that the intrathecal delivery or reduced transduction efficiency of adult peripheral neurons contributed to the reduced efficacy. Intrathecal delivery was chosen as it is the route of delivery in gene therapy trials for related disorders (6). In our study, the degree of improvement in outcomes such as NCV correlated with the extent of the knockdown of mutant Gars relative to WT Gars transcripts assayed by PCR in sensory DRGs (Supplemental Figures 7 and 8). We chose to assay DRGs because they are efficiently transduced and the sensory neurons comprise a large proportion of the tissue mass, providing the best opportunity to assess the efficiency of the knockdown. However, this analysis does not resolve this question on a cell-by-cell basis, nor does it address knockdown in motor neurons. The biodistribution of AAV9 in mice and other animals has been previously reported (37-39), and therefore, we did not repeat those analyses here. However, in pilot studies using AAV9GFP, we saw transduction in spinal cord and DRGs that closely matched published results.

Despite the unknowns regarding transduction efficiency and the extent of the knockdown, the benefit observed with post-onset delivery is encouraging. However, it is notable that we also did not see regeneration. The axon numbers did not increase when treatment was delivered after axon loss. Similarly, at NMJs, there was no evidence of collateral sprouting and reinnervation of denervated NMJs by the remaining axons. It is unclear whether this is due to insufficient knockdown of the mutant mRNA, or whether the axon degeneration is irreversible. The fact that the results were so similar in both mouse models despite the knockdown of the P278KY allele being so efficient in vitro may suggest that the degeneration is irreversible under normal circumstances. Therefore, the benefit of treatment after the onset of symptoms may be limited to slowing or stopping progression, and may need to be combined with other strategies to promote regeneration to achieve a better recovery of function.

In summary, these studies demonstrate how precision animal models can be used for testing personalized therapies for rare and orphan diseases, and provide important proof of concept for RNAi-based gene therapy for this dominant disease. This approach could be applied to other, related disorders including other dominantly inherited peripheral neuropathies or motor neuron diseases.

\section{Methods}

Clinical evaluation and mutation analysis. The proband was clinically evaluated at Texas Children's Hospital (Houston, Texas, USA). Diagnostic, whole-exome sequencing (XomeDxPlus) was performed by GeneDx (Gaithersburg, Maryland, USA). For allele-specific Sanger sequencing, we first isolated DNA from patient-derived primary fibroblasts. Cells were treated with trypsin according to the Wizard Genomic DNA Purification Kit (Promega) protocol. PCR amplification was performed to obtain a 381-bp region including GARS exon 8 using PCR SuperMix (Thermo Fisher Scientific). PCR products were cloned with the TOPO TA Cloning Kit (Thermo Fisher Scientific). Plasmid DNA from 6 isolated colonies was purified and Sanger-sequenced using the PCR primers: forward 5'-GCATTGCCAAAGTAGTACTGC-3' and reverse 5'-CCTGACTCTGATCAGTCCAGATCG-3'.

\section{GARS expression studies}

For RNA expression studies, RNA was isolated from patient fibroblasts using the RNeasy Mini Kit (Qiagen). cDNA was generated from $1 \mu \mathrm{g}$ of RNA using the High-Capacity cDNA reverse transcription kit (Applied Biosystems). The resulting cDNA was used to amplify a 224-bp product including the $\triangle \mathrm{ETAQ} G A R S$ mutation. The reaction was column purified and checked for quality via gel electrophoresis. For nextgeneration sequencing, the product was digested and "tagmented" using Tn5 transposase. The library was amplified by PCR using Kapa Hifi DNA polymerase and Illumina-compatible indexing primers. Final library fragment size and purity were determined via gel electrophoresis, and fragments were column purified and sequenced on the Illumina MiSeq with paired 155-bp reads. Overlapping reads were merged using PEAR (version 0.9.6) and aligned using BWA MEM (version 0.7.12) to custom references containing the WT exon 7/exon 8 junction or the $\triangle$ ETAQ-containing equivalent. A custom Python script (https:// github.com/kitzmanlab/gars_burgess_2019) was used to count reads with higher-scoring alignment to each junction. Uninformative reads (e.g., those not spanning the mutation) were disregarded.

For fibroblast protein expression studies, proteins were isolated in $1 \mathrm{~mL}$ cell lysis buffer $(990 \mu \mathrm{L}$ RIPA Lysis Buffer [Thermo Fisher Scientific] plus $10 \mu \mathrm{L} 100 \times$ Halt Protease Inhibitor [Thermo Fisher Scientific]) and quantified using the Pierce BCA Protein Assay Kit (Thermo Fisher Scientific). Ten micrograms of protein per sample was analyzed via Western blot. Samples were prepared in $1 \times$ SDS sample buffer (Thermo Fisher Scientific) plus $5 \mu \mathrm{L} \beta$-mercaptoethanol and boiled at $99^{\circ} \mathrm{C}$ for 10 minutes, then separated on precast 4\%-20\% Tris-glycine gels (Thermo Fisher Scientific), transferred onto a PVDF membrane, and probed with the respective primary antibody at the following dilutions in blocking solution: antiGARS 1:1000, described in ref. 17; anti-NRP1 (Abcam, EPR3113) 1:1000; and anti-actin (Sigma-Aldrich, A5060) 1:5000. Mem- 
branes were then rinsed 3 times in $1 \times$ TBST to remove unbound antibody and incubated with the respective HRP-conjugated secondary antibody at 1:10,000. Membranes were rinsed in 1× TBST and exposed using SuperSignal West Dura substrate and enhancer (Thermo Fisher Scientific).

\section{Expression construct development}

All GARS expression constructs were generated using Gateway cloning (Invitrogen). The human GARS open reading frame was amplified from human cDNA using primers with the attB1 (forward, GARS ORF $\mathrm{F}$ = ATGGACGGCGCGGGGGCTGAGG) and attB2 (reverse, GARS ORF R = TCATTCCTCGATTGTCTCT) Gateway sequences. Entry clones were generated by recombination of PCR-purified amplicons into the pDONR221 vector using BP clonase. Individual WT entry clones were confirmed by sequencing. Oligonucleotides containing sequences corresponding to each GARS mutation studied were generated, and mutagenesis was performed using the QuikChange II XL Site-Directed Mutagenesis Kit (Stratagene). Individual clones were sequenced to confirm each mutation and the absence of errors. Validated entry clones were recombined into the appropriate Gateway-compatible vector using LR clonase: pET-21a $\left({ }^{+}\right)$for aminoacylation assays and pTM3xFLAG for coimmunoprecipitation assays. For yeast complementation assays, human GARS was expressed from the pYY1 expression construct containing $\triangle \mathrm{MTS} \triangle \mathrm{WHEP} G A R S$; the pYY1 constructs were a gift from Chin-I Chien and Chien-Chia Wang (National Central University, Taoyuan City, Taiwan).

\section{Aminoacylation assays}

WT and mutant GARS proteins were expressed in E. coli with a C-terminal His tag and purified with nickel affinity resins (Novagen). The T7 transcript of human tRNA ${ }^{\text {Gly/CCC }}$ (CCC, anticodon) was prepared and purified as previously described (40), heat-denatured at $85^{\circ} \mathrm{C}$ for 3 minutes, and annealed at $37^{\circ} \mathrm{C}$ for 20 minutes before use. Steady-state aminoacylation assays were monitored at $37^{\circ} \mathrm{C}$ in $50 \mathrm{mM}$ HEPES (pH 7.5), $20 \mathrm{mM} \mathrm{KCl,} 10 \mathrm{mM} \mathrm{MgCl}, 4 \mathrm{mM}$ DTT, 2 mM ATP, and $50 \mu \mathrm{M}{ }^{3} \mathrm{H}$-glycine (PerkinElmer) at a specific activity of $16,500 \mathrm{dpm} / \mathrm{pmol}$. The reaction was initiated by mixing of GARS enzyme (20 nM for WT enzyme and $600 \mathrm{nM}$ for the $\triangle \mathrm{ETAQ}$ and P234KY mutants) with varying concentrations of tRNA $(0.3-20 \mu \mathrm{M})$. Aliquots of a reaction mixture were spotted on filter paper, quenched by $5 \%$ trichloroacetic acid, washed, dried, and measured for radioactivity using a liquid scintillation counter (LS6000SC, Beckman Coulter Inc.). The amount of radioactivity retained on filter pads was corrected for quenching effects to determine the amount of synthesis of Gly-tRNA ${ }^{\text {Gly }}$. Steady-state kinetics was determined by fitting of the initial rate of aminoacylation as a function of tRNA concentration to the Michaelis-Menten equation (41).

\section{Yeast complementation assays}

Yeast complementation assays were carried out as previously described $(17,18)$. At least 2 colonies per transformation were assayed, and each transformation was repeated at least twice.

\section{MN1 transfections for immunoprecipitation}

$\mathrm{MN} 1$ cells were grown at $37^{\circ} \mathrm{C}$ in $5 \% \mathrm{CO}_{2}$ and standard growth medium (DMEM supplemented with 10\% FBS, 2 mM L-glutamine, $100 \mathrm{U} / \mathrm{mL}$ penicillin, and $50 \mu \mathrm{g} / \mathrm{mL}$ of streptomycin [Invitrogen]). We obtained mouse MN1 cells in 2004 from Kurt Fischbeck (NIH/National Institute of Neurological Disorders and Stroke, Bethesda, Maryland, USA), who obtained them directly from the laboratory that generated these cells (H. Kim, University of Chicago, Chicago, Illinois, USA) (42). Cells were transfected using Lipofectamine 2000 (Invitrogen) with $15 \mu \mathrm{g}$ of a plasmid to express WT or mutant GARS pTM3xFLAG per T-175 flask. Cells were incubated for 48 hours at $37^{\circ} \mathrm{C}$ in $5 \% \mathrm{CO}_{2}$. Cells were then harvested using $0.25 \%$ trypsin (Invitrogen), centrifuged at $805 \mathrm{~g}$ for 2 minutes, and washed twice with $1 \times$ PBS.

\section{Coimmunoprecipitation analyses}

Twenty-five microliters of Dynabeads Protein G Magnetic beads (Invitrogen) were used for each cell lysate from one T-175 flask. Beads were washed twice in $1 \mathrm{~mL}$ of wash solution (0.5\% BSA and $0.1 \%$ Triton X-100 in PBS) and resuspended in $1 \mathrm{~mL}$ of wash solution. Two micrograms anti-FLAG antibody (Sigma-Aldrich, clone M2) was added and incubated at $4^{\circ} \mathrm{C}$ with gentle shaking overnight. Transfected cells were resuspended in $1.5 \mathrm{~mL}$ lysis buffer $(20 \mathrm{mM}$ Tris-HCl [pH 7.5], $2.5 \mathrm{mM} \mathrm{MgCl}_{2}, 300 \mathrm{mM} \mathrm{KCl,} \mathrm{0.1 \%} \mathrm{NP-40,} 1 \mathrm{mM}$ DTT, 0.2 mM PMSF, $1 \times$ Halt Protease Inhibitor Cocktail [EDTA free] [Thermo Fisher Scientific]) and incubated with gentle rocking at $4^{\circ} \mathrm{C}$ for 90 minutes. Cell lysates were centrifuged at 17,800 $g$ for 5 minutes at $4^{\circ} \mathrm{C}$. The supernatant was removed and used as the input for the immunoprecipitation (IP) reaction. Protein was quantified using the Pierce BCA Protein Assay Kit (Thermo Fisher Scientific), and $1 \mathrm{mg}$ of protein was used as input. Expression of 3xFLAG-tagged protein in input samples was confirmed by anti-FLAG M2 Western blotting. The $\alpha$ FLAG-conjugated magnetic beads were washed twice with $500 \mu \mathrm{L}$ of lysis buffer, resuspended in $25 \mu \mathrm{L}$ lysis buffer, and added to the protein sample. After 2 hours of incubation at $4^{\circ} \mathrm{C}$ with gentle shaking, beads were magnetically isolated, washed 5 times with $1 \mathrm{~mL}$ of buffer (20 mM Tris- $\mathrm{HCl}$ [pH 7.5], $2.5 \mathrm{mM} \mathrm{MgCl}_{2}, 300 \mathrm{mM} \mathrm{KCl}, 0.1 \% \mathrm{NP}-40$, $1 \mathrm{mM}$ DTT), and resuspended in $30-50 \mu \mathrm{L}$ of wash buffer per $25 \mu \mathrm{L}$ of starting beads. Two hundred micrograms per milliliter of 3xFLAG peptide was added to each sample and incubated at $4^{\circ} \mathrm{C}$ with gentle shaking for 3 hours to elute the FLAG-tagged protein.

\section{Mass spectrometry and bioinformatics}

For mass spectrometry at the Fred Hutchinson Cancer Institute, IP products were separated on a denaturing 4\%-20\% Tris-glycine gel and silver-stained using standard protocols. Destained gel slices were used for in-gel digestion with an ice-cold solution of $12.5 \mathrm{ng} / \mu \mathrm{L}$ trypsin in $50 \mathrm{mM}$ ammonium bicarbonate on ice for 1 hour. The trypsin solution was removed and replaced with $50 \mathrm{mM}$ ammonium bicarbonate, and digestion was carried out overnight at $37^{\circ} \mathrm{C}$. Peptides were extracted by the addition of $0.1 \%$ trifluoroacetic acid and light vortexing for 30 minutes followed by addition of an equal volume of $100 \%$ acetonitrile and light vortexing for an additional 30 minutes. Desalted samples were brought up in $10 \mu \mathrm{L}$ of $2 \%$ acetonitrile in $0.1 \%$ formic acid, and 8 $\mu \mathrm{L}$ was analyzed by liquid chromatography/electrospray ionization tandem mass spectrometry (MS/MS) using an Easy-nLC 1000 (Thermo Fisher Scientific) HPLC system coupled to a hybrid Orbitrap Elite (Thermo Fisher Scientific) as previously described (43). The Orbitrap Elite instrument was operated in the data-dependent mode, switching automatically between mass spectrometric survey scans in the Orbitrap with MS/MS spectra acquisition in the linear ion trap. The 15 most intense ions from the Fourier transform full scan were selected 
for fragmentation in the linear trap by collision-induced dissociation with normalized collision energy of $35 \%$. Selected ions were dynamically excluded for 30 seconds with a list size of 500 and exclusion mass width of $+/-10$ ppm.

For mass spectrometry at the University of Michigan (Ann Arbor, Michigan, USA), IP products were concentrated using TCA precipitation. Two microliters of $2 \%$ sodium deoxycholate was added to $200 \mu \mathrm{L}$ of IP product and incubated on ice for 30 minutes. Twenty microliters of $100 \%$ (wt/vol) TCA was added (bringing TCA to $10 \%$ ) and incubated on ice for 1 hour. The product was then centrifuged at maximum speed at $4^{\circ} \mathrm{C}$ for 10 minutes, and the pellet was washed in $500 \mu \mathrm{L}$ of acetone and incubated on ice for 10 minutes. The acetone solution was centrifuged at maximum speed at $4^{\circ} \mathrm{C}$ for 10 minutes. The resulting pellet was resuspended in $30 \mu \mathrm{L}$ of $20 \mathrm{mM}$ HEPES and $8 \mathrm{M}$ urea ( $\mathrm{pH}$ 8.0). Three independently generated IP products for each WT, $\triangle \mathrm{ETAQ}$, and an untagged WT GARS sample were analyzed. MS/MS was performed using the Orbitrap Fusion Mass Spectrometer (Thermo Fisher Scientific).

For all mass spectrometry data analyses, the open-source proteomics search engine X! Tandem (The Global Proteome Machine Organization) was used to match mass spectrometry data with peptide spectra. Peptide Prophet was used to validate peptide assignments (43), and Protein Prophet grouped peptides into proteins (44). ABACUS extracted spectral counts for quantitative analysis (45). Finally, interactions were scored to remove background, and comparisons of interacting proteins were performed to calculate the relative fold changes for interactions between WT and $\triangle$ ETAQ GARS.

\section{Targeted assessment of NRP1-GARS interactions}

NSC-34 cells (ATCC) were grown to 70\% confluence before transfection. Human WT, P234KY, or $\triangle \mathrm{ETAQ}$ GARS cDNAs were subcloned into the pcDNA6 plasmid in-frame with a V5 tag. Transfections were performed using Lipofectamine 2000 (Invitrogen). Thirty-six hours after transfection, cells were washed twice in PBS, scraped into PBS, pelleted, and resuspended in Pierce IP Lysis Buffer (Thermo Fisher Scientific) for 30 minutes and centrifuged for 7 minutes at 12,000 $g$; the insoluble fraction was discarded. Protein $G$ beads (Invitrogen) were preincubated with anti-NRP1 antibody (Abcam, EPR3113) or rabbit IgG (Cell Signaling Technology, 2729) for 30 minutes and then mixed with the cell lysates for overnight. Beads were then washed 3 times with buffer (100 $\mathrm{mM} \mathrm{NaCl}, 50 \mathrm{mM}$ Tris [pH 7.5], 0.1\% Triton $\mathrm{X}-100,5 \%$ glycerol). The immunoprecipitates were fractionated by 4\%-12\% Bis-Tris-Plus SDS-PAGE gels (Invitrogen) and transferred to PVDF membranes. Membranes were blocked for 1 hour with Tris-buffered saline with Tween-20 (TBST) containing 5\% nonfat dry milk. Proteins were detected using mouse monoclonal V5 antibody (Invitrogen, R960-25). NRP1 was detected using the same antibody for coimmunoprecipitation. After incubation with primary antibodies, membranes were washed and incubated with HRP-conjugated antimouse or anti-rabbit secondary antibodies (Cell Signaling Technology, 7076, 7074, respectively) followed by detection using ECL substrate (Thermo Fisher Scientific), and exposed using the FluorChem M imager (ProteinSimple).

\section{Cloning of allele-specific, mutant GARS-targeted miRNAs}

All design rules for artificial miRNAs are previously described (46), including: 22-nucleotide mature miRNA length, antisense comple- mentarity to the target (Gars; GARS), less than 60\% GC content of the mature duplex, and guide-strand biasing, such that the last 4 nucleotides of the antisense $5^{\prime}$ end were A: $\mathrm{U}$ rich, and the last 4 nucleotides of the antisense $3^{\prime}$ end were G:C rich. Mutant GARS/Gars-targeting miRNA constructs were designed with the seed match region targeting the differing nucleotides present in the mutant Gars alleles and intentional mismatches with WT GARS/Gars. MiRNAs were cloned into a U6T6 expression vector (31). After in vitro testing, lead candidate U6.miRNAs were cloned into a self-complementary proviral AAV plasmid containing a CMV-driven eGFP reporter. scAAV9 viruses were generated and titered by the Viral Vector Core at The Research Institute at Nationwide Children's Hospital (Columbus, Ohio, USA).

\section{Luciferase assays}

The dual-luciferase plasmids were created in the Psicheck2 vector (Promega), with firefly luciferase serving as a control, and the various Gars target regions cloned downstream of the Renilla luciferase stop codon. HEK293 cells were cotransfected (Lipofectamine 2000, Invitrogen) with the appropriate reporter and an individual U6.miRNA expression plasmid in a 1:5 molar ratio. GARS silencing was determined 24 hours after transfection, using the Dual-Luciferase Reporter Assay System (Promega). Triplicate data were averaged, and knockdown significance was analyzed by 2-way ANOVA. Results are presented as the mean ratio of Renilla to firefly \pm SEM.

\section{Mice}

Mice were housed in the research animal facility at The Jackson Laboratory and provided with food and water ad libitum. Gars (CAST;B6Gars $^{\mathrm{Nmf249}} / \mathrm{Rwb}$; referred to as $\operatorname{Gars}^{\mathrm{P278KY/+})}$ are previously described (22). The official strain designations of the newly engineered mouse models are B6;FVB-Gars $<$ em1Rwb $>/$ Rwb (referred to as Gars ${ }^{\text {huEx } x}$ ) and B6;FVB-Gars<em2Rwb>/Rwb (referred to as Gars ${ }^{A E T A Q /+}$ ). Experimental cohorts used for direct comparisons consisted of littermates.

\section{Generation of Gars ${ }^{\Delta E T A Q /+}$ ) and Gars $\left.^{+/ h u E \times 8}\right)$ models with CRISPR/Cas9 genome-editing technology}

Donor constructs. For Gars ${ }^{h u E x 8 /+}$, the mouse exon 8 sequence was replaced with a donor vector containing the human exon 8 sequence. The donor was a $10-\mathrm{kb}$ sequence containing a $2.8-\mathrm{kb} 5^{\prime}$ arm of homology and a 7-kb $3^{\prime}$ arm of homology isolated from a C57BL/6J BAC library flanking the human exon 8 sequence. For Gars $^{4 E T A Q /+}$, the donor construct consisted of an single-stranded oligonucleotide sequence spanning the first 52 bases of mouse exon 8 with short arms of homology (see below for sequence) containing a 12-base deletion (bases 12-23 of exon 8).

Microinjection. Preparation and microinjection of CRISPR/ Cas9 reagents were performed as previously described (47). All components, including Cas9 mRNA (100 ng/ $\mu \mathrm{L}$; either TriLink or synthesized by in vitro transcription), sgRNA, guides 144 and 1340 (50 ng/ $\mu \mathrm{L}$; guide sequence below), and each donor vector (20 ng/ $\mu \mathrm{L}$ plasmid DNA or $100 \mathrm{ng} / \mu \mathrm{L}$ single-stranded donor oligonucleotides), were injected into the male pronucleus and cytoplasm of about 300 zygotes at the pronuclei stage. All zygotes were isolated from superovulated FVB/NJ (JAX stock 001600, The Jackson Laboratory) females mated with C57BL/6NJ (JAX stock 005304) males. Then, groups of 15-25 blastocysts were transferred into the uterus of pseudopregnant females. 
The ssODN donor sequence was AGTTTACTTGTAACAGGCTTTGTTTTATTGGAAGCACATTGTCTTACTTGTAATAGACTGGTTTATTTAATTTTATAGATACTTGAGACCGGGGATTTTCtTGAATTTCAAACGACTTTTGGAATTCAAC. The sgRNA 144 sequence was AAAATTCCCTGTGCAGTTTC. The sgRNA 1340 sequence was TCAGAAATGAGATCTCACCT.

Genotyping. Transgenic mice were genotyped for the presence of either the humanized exon 8 or $\triangle$ ETAQ constructs. Genomic DNA was prepared from tail biopsy lysed with proteinase K. Primers HuEx8FO_F:CATAACATCACGCGTGGTTCC and HuEx8RO_R:CAAGTGTGGCGGTTTCCATC that span the 2.8-kb 5' arm of homology to the $3^{\prime}$ end of Gars exon 8 and subsequent Sanger sequencing with HuEx8RO_R were used to identify human single-nucleotide polymorphisms in exon 8 of Gars within Gars ${ }^{h u E x s}$ founders and subsequent generations. Primers $\triangle E T A Q F O F$ :GGCCATAAGCATAATTTTACTGTG and $\triangle$ ETAQFO_R:TACAACAGAAACAAACTGTGGTCA with subsequent Sanger sequencing with $\triangle$ ETAQFO_R were used to detect the 12-bp deletion in bases 13-24 in Gars $^{\triangle E T A Q /+}$ founders and subsequent generations.

Reverse transcriptase PCR. Primers Gars2F_CTCCCACCACTGGCAATGAC and Gars2R_CTCACTCAGCAGCAGCTCC were used to amplify a portion of the Gars open reading frame spanning Gars exon 8 from first-strand cDNA generated from sciatic nerve RNA isolated from Gars $^{+/ h u E x 8}$ and Gars $^{4 E T A Q / h u E x 8}$ mice. Humanized exon 8 and $\triangle$ ETAQ transcript sequences were identified with Sanger sequencing and primer Gars $2 \mathrm{~F}$.

Tissue lysate preparation. Whole brain samples were isolated from animals immediately after they were euthanized by $\mathrm{CO}_{2}$ inhalation. The tissues were frozen in liquid nitrogen and stored at $-80^{\circ} \mathrm{C}$. Samples were homogenized using a mortar and pestle followed by a Dounce homogenizer in 1\% NP-40 in PBS supplemented with Protease Inhibitor Cocktail Tablets (Roche), then centrifuged at 14,000 $\mathrm{g}$ twice for 5 minutes at $4^{\circ} \mathrm{C}$. Cleared homogenates were then sonicated at $4^{\circ} \mathrm{C}$ and centrifuged again at $14,000 \mathrm{~g}$ for 5 minutes. Twenty micrograms of protein was then analyzed by immunoblot.

Western blot analysis. Protein lysates were resolved on MiniPROTEAN 4\%-15\% Tris-glycine gels (Bio-Rad) and transferred to an Invitrolon \& Immobilon-P PVDF membrane for Western blot analysis. Membranes were blocked with $5 \%$ skim milk in TBST (1× Tris-buffered saline, 0.1\% Tween-20), and incubated overnight with anti-GARS (rabbit; Abcam, ab42905; 1:1000 dilution) and anti-NeuN (mouse monoclonal; Cell Signaling Technology, E4M5P; 1:1000) diluted in blocking solution at $4^{\circ} \mathrm{C}$. After three 10-minute washes in TBST, the blots were incubated with the appropriate HRP-conjugated secondary antibodies (PerkinElmer, NEF812, NEF822001EA) diluted in blocking solution. After three 10-minute washes in TBST, the blots were developed using Western Lightening Plus-ECL, Enhanced Chemiluminescence Substrate (PerkinElmer).

\section{Assessment of axonal neuropathy in all mouse models}

Grip strength was evaluated by wire hang test $(20,26)$ to evaluate gross muscle strength and endurance. Nerve conduction studies, motor nerve histology and analysis, NMJ immunofluorescence and analysis, and body weight evaluation were completed as previously described $(20,30)$. These studies were not formally blinded. First, the Gars mice have a visible phenotype that makes blinding with respect to genotype difficult, although mice treated at birth were often rescued to an extent that made them difficult to distinguish from control mice. The primary evaluator of the in vivo mouse studies was also the only person proficient at the intrathecal and i.c.v. injections at the time. Therefore, although records of which mice were treated or untreated were not immediately at hand during the analysis, this information was available to the tester.

\section{Neonatal delivery of scAAV9.mi.P278KY and scAAV9.mi.AETAQ}

Before all injections of mice at P0-P1, all pups were anesthetized via cryoanesthesia as previously described (48). Then, all i.c.v. injections were performed using a Hamilton syringe (catalog 65460_03) with a 32-gauge needle and escalating doses of scAAV9.mi.P278KY as stated in Supplemental Tables 2 and 3 or $2.6 \times 10^{11}$ DNAse-1-resistant particles (DRPS) per mouse of mi. $\triangle$ ETAQ (about 2-10 $\mu \mathrm{L}$ ) diluted in sterile PBS. All gene therapy vectors were injected in to the lateral ventricles by positioning of the needle directly lateral to the sagittal suture and rostral to the neonatal coronal suture. For i.v. injections, all cryoanesthetized mice were injected with $1 \times 10^{11}$ DRPS per mouse directly into the superficial temporal vein in a caudal orientation with the use of a Hamilton syringe (catalog 7655-01) with a 32-gauge needle.

\section{Intrathecal delivery of gene therapy constructs to post-onset mice}

With the use of a Hamilton syringe (catalog 7655-01) with a 32-gauge needle, all adult post-onset mice were injected with approximately 1 $\times 10^{11}$ DRPS per mouse of scAAV9.mi.P278KY or scAAV9.mi. $\mathrm{ETAQ}$ diluted into sterile PBS $(\sim 10 \mu \mathrm{L})$ via an intrathecal injection by lumbar puncture. Here, all mice were anesthetized with isoflurane and received an injection of the proper vector into the $\mathrm{L} 6$ spinous process with the use of a Hamilton syringe with a 32-gauge needle. Each vector was slowly injected and the needle left in place for 5-10 seconds before withdrawal.

\section{Quantification of allele-specific expression}

Whole liver and lumbar DRG samples were isolated from animals immediately after they were euthanized by cervical dislocation. The tissues were frozen in liquid nitrogen and stored at $-80^{\circ} \mathrm{C}$. Samples were homogenized using a mortar and pestle followed by a Dounce homogenizer, and RNA was isolated from liver using Trizol Reagent (catalog 15596018, Thermo Fisher Scientific) and DRGs using either an RNeasy Mini Kit (catalog 74104 and 74106, Qiagen) or a mirVana miRNA Isolation Kit (catalog AM1560, Thermo Fisher Scientific). All RNA samples were reverse transcribed using SuperScript III First-Strand Synthesis System (catalog 18080051). To quantify allele-specific expression of WT and mutant GARS, EpigenDx performed pyrosequencing on the PSQ96 HS System (Qiagen) following the manufacturer's instructions, using custom assays.

\section{Statistics}

Statistical tests were performed using GraphPad Prism 7 software. A 2-tailed Student's $t$ test or 1-way or 2-way ANOVA followed by Tukey's honestly significant difference (HSD) post hoc comparisons test (as indicated in the figure legends) was used to determine significant differences between treatment groups and/or genotypes for axon counts, conduction velocity, grip strength, and body weight. Axon diameters were compared using nonparametric Kolmogorov-Smirnov 2-sample and Shapiro-Wilk normality tests. NMJ innervation status between genotypes and categories (fully innervated, partially innervated, and denervated) was evaluated 
with a 2-way ANOVA followed by Tukey's HSD post hoc comparisons test. A $P$ value less than 0.05 was considered significant.

\section{Study approval}

Clinical procedures and sequencing that went beyond standard-ofcare clinical evaluation and diagnostics were performed under the Institutional Review Board-approved protocol H-29697, Genome Sequencing to Elucidate the Causes and Mechanisms of Mendelian Genetic Disorders, Baylor College of Medicine, Houston, Texas, USA. Clinical data were obtained after written informed consent from the proband's parents. All mouse husbandry and experimental procedures were conducted according to the NIH Guide for the Care and Use of Laboratory Animals (National Academies Press, 2011) and were reviewed and approved by the Animal Care and Use Committee of The Jackson Laboratory, Bar Harbor, Maine, under Animal Use Summary 1026, "The formation and maintenance of neuronal synapses and circuits."

\section{Author contributions}

$\mathrm{RWB}, \mathrm{SQH}$, and AA contributed to experimental design, data interpretation, and manuscript preparation, with input from all authors. In vivo studies were completed by KHM; additional in vivo studies were conducted by TJH and ELS. All miGARS constructs were designed by $\mathrm{SQH}$ and constructed and tested for efficacy in vitro by NKP. AMF assisted with viral vector production. RNA isolation and cDNA synthesis from mouse tissue were completed by SGK, NKP, LMW, and KHM. Neuropilin IP was performed by NW and XLY. Enzyme kinetics were evaluated by RT and YMH. Yeast complementation studies and evaluation of human $\triangle$ ETAQ expression were done by LBG and SNO. Patient clinical, pathological, and genomic evaluations were done by AN, JRL, PM, IJB, JOK, DM, and RMS.

\section{Acknowledgments}

The authors are indebted to the patient and her family for their participation and outstanding support for the study. We would also like to thank Chin-l Chien and Chien-Chia Wang for the pYY1 expression construct, Stephanie Bielas for anonymized control fibroblast cells, and the Viral Vector Core Facility at Nation- wide Children's Hospital for AAV9 production. At The Jackson Laboratory, Bonnie Lyons, Rosilinda Dotti, Kathy Miers, Peter Finger, and the In Vivo Pharmacology Service provided training, consultation, technical assistance, and infrastructure. RWB is supported by the NIH (NS054154, OD020351, NS105116), the Muscular Dystrophy Association (MDA 351564), and the Director's Innovation Fund of The Jackson Laboratory. KHM is supported by an NIH predoctoral fellowship (NSO98540), and ELS is supported by an NIH predoctoral fellowship (NS100328). AA is supported by a grant from the National Institute of General Medical Sciences (GM118647) and by the Muscular Dystrophy Association (MDA 294479). LBG was supported by an NIH Cellular and Molecular Biology Training Grant (GM007315), an NIH Medical Scientist Training Grant (GM07863), and an NIH F30 National Research Service Award (NS092238). RM is supported by the Michigan Predoctoral Training in Genetics Program (GM007544). SQH is supported by grants from the NIH (National Institute of Arthritis and Musculoskeletal and Skin Diseases [NIAMS] R01AR062123; NIAMS Center of Research Translation in Muscular Dystrophy Therapeutic Development grant 1P50AR070604; and National Institute of Neurological Disorders and Stroke [NINDS] R21NS101166) and the Muscular Dystrophy Association (MDA 351564). JRL is supported in part by grants from the NINDS (R01NS058529 and R35NS105078) and a joint National Human Genome Research Institute/National Heart, Lung, and Blood Institute grant (UM1HG006542) to the Baylor Hopkins Center for Mendelian Genomics. The scientific services at The Jackson Laboratory are supported by NIH grant CA034196.

Address correspondence to: Robert W. Burgess, The Jackson Laboratory, 600 Main Street, Bar Harbor, Maine 04609, USA. Phone: 207.288.6706; Email: Robert.Burgess@jax.org. Or to: Scott Q. Harper, The Research Institute at Nationwide Children's Hospital, 700 Children's Drive, Columbus, Ohio 43205, USA. Phone: 614.355.2893; Email: Scott.Harper@nationwidechildrens. org. Or to: Anthony Antonellis, Department of Human Genetics, University of Michigan, 3710A Med Sci II, 1241 E. Catherine Street SPC 5619, Ann Arbor, Michigan 48109-5618, USA. Phone: 734.647.4058; Email: antonell@umich.edu.
1. Capdeville R, Buchdunger E, Zimmermann J, Matter A. Glivec (STI571, imatinib), a rationally developed, targeted anticancer drug. Nat Rev Drug Discov. 2002;1(7):493-502.

2. Reck M, et al. Pembrolizumab versus chemotherapy for PD-L1-positive non-small-cell lung cancer. N Engl J Med. 2016;375(19):1823-1833.

3. Chong JX, et al. The genetic basis of mendelian phenotypes: discoveries, challenges, and opportunities. Am J Hum Genet. 2015;97(2):199-215.

4. Robinson PN, et al. Improved exome prioritization of disease genes through cross-species phenotype comparison. Genome Res. 2014;24(2):340-348.

5. Naldini L. Gene therapy returns to centre stage. Nature. 2015;526(7573):351-360.

6. Mendell JR, et al. Single-dose gene-replacement therapy for spinal muscular atrophy. N EnglJ Med. 2017;377(18):1713-1722.
7. Bainbridge JW, et al. Long-term effect of gene therapy on Leber's congenital amaurosis. N Engl J Med. 2015;372(20):1887-1897.

8. Saporta MA, Shy ME. Inherited peripheral neuropathies. Neurol Clin. 2013;31(2):597-619.

9. Boylan K. Familial amyotrophic lateral sclerosis. Neurol Clin. 2015;33(4):807-830.

10. Antonellis A, et al. Glycyl tRNA synthetase mutations in Charcot-Marie-Tooth disease type 2D and distal spinal muscular atrophy type V. Am J Hum Genet. 2003;72(5):1293-1299.

11. Sivakumar K, et al. Phenotypic spectrum of disorders associated with glycyl-tRNA synthetase mutations. Brain. 2005;128(pt 10):2304-2314.

12. Wei N, Zhang Q, Yang XL. Neurodegenerative Charcot-Marie-Tooth disease as a case study to decipher novel functions of aminoacyl-tRNA synthetases. J Biol Chem. 2019;294(14):5321-5339.

13. Abe A, Hayasaka K. The GARS gene is rarely mutated in Japanese patients with Charcot-
Marie-Tooth neuropathy. J Hum Genet 2009;54(5):310-312.

14. James PA, Cader MZ, Muntoni F, Childs AM, Crow YJ, Talbot K. Severe childhood SMA and axonal CMT due to anticodon binding domain mutations in the GARS gene. Neurology. 2006;67(9):1710-1712.

15. Lee HJ, et al. Two novel mutations of GARS in Korean families with distal hereditary motor neuropathy type V. J Peripher Nerv Syst. 2012;17(4):418-421.

16. Rohkamm B, et al. Further evidence for genetic heterogeneity of distal HMN type V, CMT2 with predominant hand involvement and Silver syndrome. J Neurol Sci. 2007;263(1-2):100-106.

17. Antonellis A, et al. Functional analyses of glycyltRNA synthetase mutations suggest a key role for tRNA-charging enzymes in peripheral axons. JNeurosci. 2006;26(41):10397-10406.

18. Griffin LB, et al. Impaired function is a common 
feature of neuropathy-associated glycyltRNA synthetase mutations. Hum Mutat. 2014;35(11):1363-1371.

19. Oprescu SN, et al. Compound heterozygosity for loss-of-function GARS variants results in a multisystem developmental syndrome that includes severe growth retardation. Hum Mutat. 2017;38(10):1412-1420.

20. Motley WW, et al. Charcot-Marie-Tooth-linked mutant GARS is toxic to peripheral neurons independent of wild-type GARS levels. PLoS Genet. 2011;7(12):e1002399.

21. Lek M, et al. Analysis of protein-coding genetic variation in 60,706 humans. Nature. 2016;536(7616):285-291.

22. Seburn KL, Nangle LA, Cox GA, Schimmel P, Burgess RW. An active dominant mutation of glycyl-tRNA synthetase causes neuropathy in a Charcot-Marie-Tooth 2D mouse model. Neuron. 2006;51(6):715-726.

23. He W, et al. CMT2D neuropathy is linked to the neomorphic binding activity of glycyl-tRNA synthetase. Nature. 2015;526(7575):710-714.

24. Posey JE, et al. Resolution of disease phenotypes resulting from multilocus genomic variation. N Engl JMed. 2017;376(1):21-31.

25. Qin X, Hao Z, Tian Q, Zhang Z, Zhou C, Xie W. Cocrystal structures of glycyl-tRNA synthetase in complex with tRNA suggest multiple conformational states in glycylation. J Biol Chem. 2014;289(29):20359-20369.

26. Achilli F, et al. An ENU-induced mutation in mouse glycyl-tRNA synthetase (GARS) causes peripheral sensory and motor phenotypes creating a model of Charcot-Marie-Tooth type 2D peripheral neuropathy. Dis Model Mech. 2009;2(7-8):359-373.

27. Sleigh JN, Grice SJ, Burgess RW, Talbot K, Cader MZ. Neuromuscular junction maturation defects precede impaired lower motor neuron connectivity in Charcot-Marie-Tooth type 2D mice. Hum Mol Genet. 2014;23(10):2639-2650.

28. Spaulding EL, Sleigh JN, Morelli KH, Pinter MJ,
Burgess RW, Seburn KL. Synaptic deficits at neuromuscular junctions in two mouse models of Charcot-Marie-Tooth type 2d. J Neurosci. 2016;36(11):3254-3267.

29. Bais P, et al. Metabolite profile of a mouse model of Charcot-Marie-Tooth type 2D neuropathy: implications for disease mechanisms and interventions. Biol Open. 2016;5(7):908-920.

30. Morelli KH, et al. Severity of demyelinating and axonal neuropathy mouse models is modified by genes affecting structure and function of peripheral nodes. Cell Rep. 2017;18(13):3178-3191.

31. Boudreau R, Garwick-Coppens S, Liu J, Wallace L, Harper S. Rapid cloning and validation of microRNA shuttle vectors: a practical guide. In: Harper SQ, ed. RNA Interference Techniques. New York, New York, USA: Humana Press; 2011:19-37.

32. Niehues S, et al. Impaired protein translation in Drosophila models for Charcot-Marie-Tooth neuropathy caused by mutant tRNA synthetases. Nat Commun. 2015;6:7520.

33. Miller VM, et al. Allele-specific silencing of dominant disease genes. Proc Natl Acad Sci U S A. 2003;100(12):7195-7200.

34. Miller VM, Gouvion CM, Davidson BL, Paulson HL. Targeting Alzheimer's disease genes with RNA interference: an efficient strategy for silencing mutant alleles. Nucleic Acids Res. 2004;32(2):661-668.

35. Rodriguez-Lebron E, Paulson HL. Allele-specific RNA interference for neurological disease. Gene Ther. 2006;13(6):576-581.

36. Li C, Xiao P, Gray SJ, Weinberg MS, Samulski RJ. Combination therapy utilizing shRNA knockdown and an optimized resistant transgene for rescue of diseases caused by misfolded proteins. Proc Natl Acad Sci U S A. 2011;108(34):14258-14263.

37. Snyder BR, et al. Comparison of adenoassociated viral vector serotypes for spinal cord and motor neuron gene delivery. Hum Gene Ther. 2011;22(9):1129-1135.

38. Schuster DJ, et al. Biodistribution of adeno- associated virus serotype 9 (AAV9) vector after intrathecal and intravenous delivery in mouse. Front Neuroanat. 2014;8:42.

39. Meyer K, et al. Improving single injection CSF delivery of AAV9-mediated gene therapy for SMA: a dose-response study in mice and nonhuman primates. Mol Ther. 2015;23(3):477-487.

40. Hou YM, Westhof E, Giegé R. An unusual RNA tertiary interaction has a role for the specific aminoacylation of a transfer RNA. Proc Natl Acad Sci U S A. 1993;90(14):6776-6780.

41. Schreier AA, Schimmel PR. Transfer ribonucleic acid synthetase catalyzed deacylation of aminoacyl transfer ribonucleic acid in the absence of adenosine monophosphate and pyrophosphate. Biochemistry. 1972;11(9):1582-1589.

42. Salazar-Grueso EF, Kim S, Kim H. Embryonic mouse spinal cord motor neuron hybrid cells. Neuroreport. 1991;2(9):505-508.

43. Keller A, Nesvizhskii AI, Kolker E, Aebersold R. Empirical statistical model to estimate the accuracy of peptide identifications made by MS/MS and database search. Anal Chem. 2002;74(20):5383-5392.

44. Nesvizhskii AI, Keller A, Kolker E, Aebersold R. A statistical model for identifying proteins by tandem mass spectrometry. Anal Chem. 2003;75(17):4646-4658.

45. Fermin D, Basrur V, Yocum AK, Nesvizhskii AI. Abacus: a computational tool for extracting and pre-processing spectral count data for label-free quantitative proteomic analysis. Proteomics. 2011;11(7):1340-1345.

46. Wallace LM, et al. Pre-clinical safety and offtarget studies to support translation of AAVmediated RNAi therapy for FSHD. Mol Ther Methods Clin Dev. 2018;8:121-130.

47. Qin W, et al. Generating mouse models using CRISPR-Cas9-mediated genome editing. Curr Protoc Mouse Biol. 2016;6(1):39-66.

48. Phifer CB, Terry LM. Use of hypothermia for general anesthesia in preweanling rodents. Physiol Behav. 1986;38(6):887-890. 